\title{
ADINKRAS FOR MATHEMATICIANS
}

\author{
YAN X. ZHANG
}

\begin{abstract}
Adinkras are graphical tools created to study representations of supersymmetry algebras. Besides having inherent interest for physicists, the study of adinkras has already shown non-trivial connections with coding theory and Clifford algebras. Furthermore, adinkras offer many easy-to-state and accessible mathematical problems of algebraic, combinatorial, and computational nature. We survey these topics for a mathematical audience, make new connections to other areas (homological algebra and poset theory), and solve some of these said problems, including the enumeration of all hypercube adinkras up through dimension 5 and the enumeration of odd dashings of adinkras for any dimension.
\end{abstract}

\section{INTRODUCTION}

In a series of papers starting with [11, different subsets of the "DFGHILM collaboration" (Doran, Faux, Gates, Hübsch, Iga, Landweber, Miller) have built and extended the machinery of adinkras. Following the ubiquitous spirit of visual diagrams in physics, adinkras are combinatorial objects that encode information about the representation theory of supersymmetry algebras. Adinkras have many intricate links with other fields such as graph theory, Clifford theory, and coding theory. Each of these connections provide many problems that can be compactly communicated to a (non-specialist) mathematician. This paper is a humble attempt to bridge the language gap and generate communication.

In short, adinkras are chromotopologies (a class of edge-colored bipartite graphs) with two additional structures, a dashing condition on the edges and a ranking condition on the vertices. We redevelop the foundations in a self-contained manner in Sections 2 and 4, leading to a cleanly-nested set of classifications (Theorems 4.3 , 4.4. and 4.5).

Using this setup, we look at the two aforementioned conditions separately in Sections 5 and 6. In each of these sections we make an original connection with a different area of mathematics and generalize the corresponding notion to wider classes of graphs. In Section 5 we use homological algebra to study dashings; our main result is the enumeration of odd dashings for any chromotopology. In Section [6, we use the theory of posets to put a lattice structure on the set of all rankings of any bipartite graph (including chromotopologies); we also count hypercube rankings up through dimension 5 .

While we wish that the reader would enjoy the motivation from physics sketched out in Section 3. this section may be skipped for someone only interested in mathematical problems. However, we hope this paper will allow the reader to better

Received by the editors May 11, 2012 and, in revised form, November 22, 2012.

2010 Mathematics Subject Classification. Primary 05A99.

(C) 2014 American Mathematical Society Reverts to public domain 28 years from publication 
appreciate the foundational problems of adinkras from the physics literature. We return to these roots in Section 7 , ending with a quick survey of recent developments and some original observations.

\section{Definitions}

We assume basic notions of graphs. For a graph $G$, we use $E(G)$ to denote the edges of $G$ and $V(G)$ to denote the vertices of $G$. We also assume most basic notions of posets (there are many references, including [25]).

One slight deviation from the literature is that we consider the Hasse diagram for a poset as a directed graph, with $x \rightarrow y$ an edge if $y$ covers $x$. Thus it makes sense to call the maximal elements (i.e. those $x$ with no $y>x$ ) sinks and the minimal elements sources.

For this paper, a ranked poset is a poset $A$ equipped with a rank function $h: A \rightarrow$ $\mathbf{Z}$ such that for all $x$ covering $y$ we have $h(x)=h(y)+1$. There is a unique rank function $h_{0}$ among these such that 0 is the lowest value in the range of $h_{0}$, so it makes sense to define the rank of an element $v$ as $h_{0}(v)$. The largest element in the range of $h_{0}$ is then the length of the longest chain in $A$; we call it the height of $A$. We remark that such a poset is often called a graded poset, though there are similar but subtly different uses of that name. Thus, we use ranked to avoid ambiguity.

2.1. Chromotopologies and adinkras. An $n$-dimensional chromotopology is a finite connected simple graph $A$ such that the following conditions hold:

- $A$ is $n$-regular (every vertex has exactly $n$ incident edges) and bipartite. Respecting the physics literature, we call the two sets in the bipartition of $V(A)$ bosons and fermions. As the actual choice is mostly arbitrary for our purposes, we will usually not explicitly include this data.

- The elements of $E(A)$ are colored by $n$ colors, which are elements of the set $[n]=\{1,2, \ldots, n\}$ unless denoted otherwise, such that every vertex is incident to exactly one edge of each color.

- For any distinct $i$ and $j$, the edges in $E(A)$ with colors $i$ and $j$ form a disjoint union of 4-cycles.

We now introduce the key example of a chromotopology in our paper. Define the $n$-dimensional hypercube $I^{n}$ to be the graph with $2^{n}$ vertices labeled by the $n$-codewords, with an edge between two vertices if they differ by exactly one bit. It is easy to see that $I^{n}$ is bipartite and $n$-regular. Noticing that $I=I^{1}$ is just a single edge, our exponentiation $I^{n}$ is justified as a cartesian product. Now, if two vertices differ at some bit $i, 1 \leq i \leq n$, color the edge between them with the color $i$. The 2-colored 4-cycle condition holds, so we get a chromotopology that we call the $n$-cubical chromotopology $I_{c}^{n}$. Figure 1 shows $I_{c}^{3}$.

We now define two structures we can put on a chromotopology.

(1) Let a ranking of a bipartite graph (in particular, any chromotopology) $A$ be a map $h: V(A) \rightarrow \mathbf{Z}$ that gives $A$ the additional structure of a ranked poset on $A$ via $h$ as the rank function. By this, we mean that we identify $A$ with the Hasse diagram of the said ranked poset with rank function $h$. We consider two rankings equivalent if they differ only by a translate, as the resulting ranked posets would then be isomorphic. Given a ranking $h$ of $A$, we say that $A$ is ranked by $h$. 


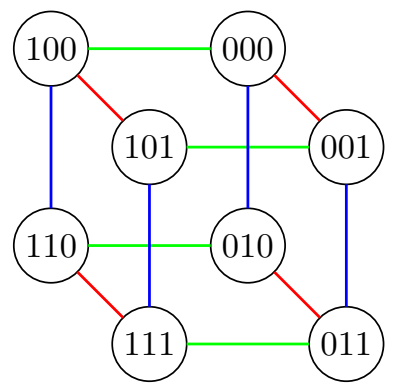

Figure 1. The 3-cubical chromotopology $I_{c}^{3}$. We can take the bosons to be either $\{000,011,101,110\}$ or $\{001,010,100,111\}$ and take the fermions to be the other set.

In this paper, such as in Figure 3, we will usually represent ranks via vertical placement, with higher values of $h$ corresponding to being higher on the page. The vertices at the odd ranks and the vertices at the even ranks naturally form the bipartition of $V(A)$.

Any bipartite graph (and thus any chromotopology) $A$ can be ranked as follows: take one choice of a bipartition of $V(A)$ into bosons and fermions. Assign the rank function $h$ to take values 0 on all bosons and 1 on all fermions, which creates a ranked poset with 2 ranks. We call the corresponding ranking a valise. Because we could have switched the roles of bosons and fermions, each bipartite graph gives rise to exactly two valises. For an example, see Figure 2 .

We remark that in the existing literature, such as [7, posets are never mentioned and the following equivalent definition is used, under the names engineerable or non-escheric: give $A$ the structure of a directed graph, such that in traversing the boundary of any (non-directed) loop with a choice of direction, the number of edges oriented along the direction equals the number of edges oriented against the direction. This is easily seen to be equivalent to our definition.

(2) Let a dashing of a bipartite graph $A$ be a map $d: E(A) \rightarrow \mathbf{Z}_{2}$. Given a dashing $d$ of $A$, we say that $A$ is dashed by $d$. We visually depict a dashing as making each edge $e \in E(A)$ either dashed or solid, corresponding to $d(e)=1$ or 0 respectively. We will slightly abuse notation and write $d(v, w)$ to mean $d((v, w))$, where $(v, w)$ is an edge from $v$ to $w$.

For a chromotopology $A$, a dashing is an odd dashing if the sum of $d(e)$ as $e$ runs over each 2-colored 4-cycle (that is, a 4-cycle of edges that use a total of 2 colors) is $1 \in \mathbf{Z}_{2}$ (alternatively, every 2-colored 4-cycle contains an odd number of dashed edges). If $A$ is dashed by an odd dashing $d$, we say that $A$ is well-dashed.

An adinkra is a ranked well-dashed chromotopology. We call a graph that can be made into an adinkra adinkraizable. Since any chromotopology, being a bipartite graph, can be ranked, adinkraizability is equivalent to the condition of having at least one odd dashing. A well-dashed chromotopology is just an adinkraizable chromotopology equipped with this dashing. 


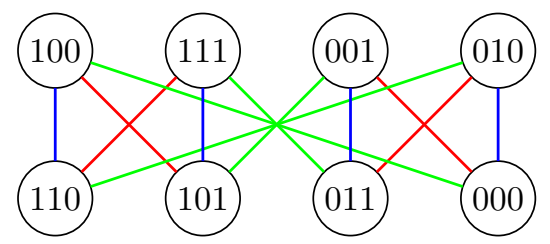

Figure 2. A valise; one possible ranking of the chromotopology $I_{c}^{3}$.

We frequently use some forgetful functions in the intuitive way: for example, given any (possibly ranked and/or well-dashed) chromotopology $A$, we will use "the chromotopology of $A$ " to mean the underlying edge-colored graph of $A$, forgetting the ranking and the dashing.

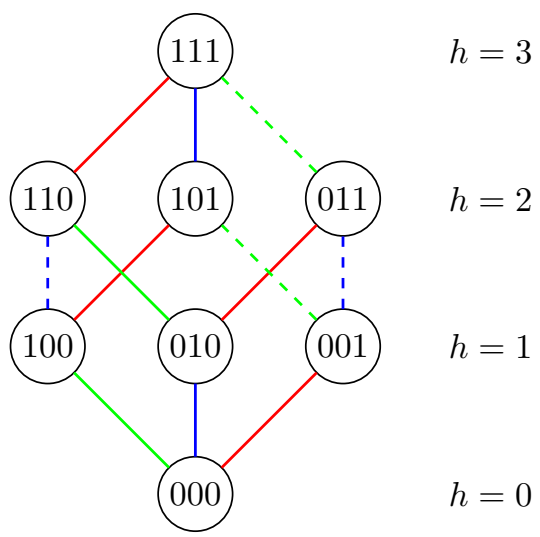

FiguRE 3. An adinkra with the chromotopology $I_{c}^{3}$.

Many of our proofs involve algebraic manipulation. To make our treatment more streamlined, we now set up algebraic interpretations of our definitions.

- The condition of $A$ being a chromotopology is equivalent to having a map $q_{i}: V(A) \rightarrow V(A)$ for every color $i$ that sends each vertex $v$ to the unique vertex connected to $v$ by the edge with color $i$, such that the different $q_{i}$ commute (equivalently, the $q_{i}$ generate a $\mathbf{Z}_{2}^{n}$ action on $V(A)$ ). The welldefinedness of the $q_{i}$ corresponds to the edge-coloring condition and the commutation requirement corresponds to the 4-cycle condition. Note that $q_{i}$ is an involution, as applying $q_{i}$ twice simply traverses the same edge twice. Furthermore, $q_{i}$ sends any boson to a fermion, and vice versa.

- The condition of a chromotopology $A$ being well-dashed (with dashing function $d$ ) is equivalent to having the maps $\bar{q}_{i}$ anticommute, where we define $\bar{q}_{i}: \mathbf{R}[V(A)] \rightarrow \mathbf{R}[V(A)]$ for every color $i$ by $\bar{q}_{i}(v)=d\left(v, q_{i}(v)\right) q_{i}(v)$.

2.2. Multigraphs. It seems natural to extend our definition to multigraphs. Let a multichromotopology be a generalization of chromotopology where we relax the condition that the graph be simple and now allow loops and multiple-edges. The $n$ regular condition remains, but is reinterpreted so that a loop counts as degree 1 as opposed to 2 . The algebraic condition is still that the $q_{i}$ must commute. However, 
the combinatorial version of the rule (that the union of edges of different colors $i$ and $j$ form a disjoint union of 4-cycles) must be extended to allow degenerate 4cycles that use double-edges or loops. Define the well-dashed and ranked properties on multichromotopologies analogously, again extending our condition for 2-colored 4-cycles to allow double-edges and loops.

These generalizations exclude each other in a cute way:

- While there are ranked multichromotopologies with double-edges, no welldashed multichromotopology can have a double-edge because a double-edge immediately gives a degenerate 2-colored 4-cycle, and it is impossible for the sum of dashes over a degenerate 2-colored 4-cycle to be even.

- The loops have the opposite problem: they allow new well-dashed multichromotopologies, but none of these multichromotopologies can be ranked because bipartite graphs cannot have loops.

See Figure 4 for some examples.

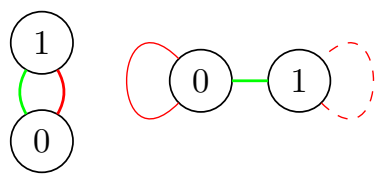

Figure 4. A ranked multichromotopology (with double-edge) that cannot be well-dashed, and a well-dashed multichromotopology (with loops) that cannot be ranked.

The above discussion shows that the natural definition of multiadinkras, that is, well-dashed ranked multichromotopologies, does not give us any new objects that are not already adinkras. However, multigraphs naturally appear in our classification paradigm in Section 4.1, so they are still a nice notion to have for this paper.

\section{Physical motivation}

The reader is equipped to understand the rest of the paper (with the exception of Section 7) with no knowledge from this section. However, we hope our brief outline will serve as enrichment that may provide some additional intuition, as well as provide a review of the original problems of interest (where much remains to be done). While knowledge of physics will help in understanding this section, it is by no means necessary. We have neither the space nor the qualification to give a comprehensive review, so we encourage interested readers to explore the original physics literature.

The physics motivation for adinkras is the following: "we want to understand offshell representations of the $N$-extended Poincaré superalgebra in the 1-dimensional worldline". There is no need to understand what all of these terms mean 1 to appreciate the rest of the discussion; we now sketch the thinking process that leads to adinkras.

\footnotetext{
${ }^{1}$ The author certainly does not.
} 
Put simply, we are looking at the representations of the algebra $\mathfrak{p o}^{1 \mid N}$ generated by $N+1$ generators $Q_{1}, Q_{2}, \ldots, Q_{N}$ (the supersymmetry generators) and $H=i \partial_{t}$ (the Hamiltonian), such that

$$
\begin{aligned}
\left\{Q_{I}, Q_{J}\right\} & =2 \delta_{I J} H, \\
{\left[Q_{I}, H\right] } & =0 .
\end{aligned}
$$

Here, $\delta$ is the Kronecker delta, $\{A, B\}=A B+B A$ is the anticommutator, and $[A, B]=A B-B A$ is the commutator. We can also say that $\mathfrak{p o}^{1 \mid N}$ is a superalgebra where the $Q_{i}$ 's are odd generators and $H$ is an even generator. Since $H$ is basically a time derivative, it changes the engineering dimension (physics units) of a function $f$ by a single power of time when acting on $f$.

Consider $\mathbf{R}$-valued functions $\left\{\phi_{1}, \ldots, \phi_{m}\right\}$ (the bosonic fields or bosons) and $\left\{\psi_{1}, \ldots, \psi_{m}\right\}$ (the fermionic fields or fermions), collectively called the component fields. The fact that the two cardinalities match comes from the physics assumption that the representations are off-shell; i.e. the component fields do not obey other differential equations. We want to understand representations of $\mathfrak{p o}^{1 \mid N}$ acting on the following infinite basis:

$$
\left\{H^{k} \phi_{I}, H^{k} \psi_{J} \mid k \in \mathbf{N} ; I, J \leq m\right\}
$$

There is a subtlety here, as these infinite-dimensional representations are frequently called "finite-dimensional" by physicists, who would just call the $\left\{\phi_{I}\right\}$ and the $\left\{\psi_{I}\right\}$ the "basis," emphasizing the finiteness of $m$. A careful treatment of this is given in [6]. A long-open problem is:

Question 3.1. What are all such "finite-dimensional" representations of $\mathfrak{p o}^{1 \mid N}$ ? What if we extend to higher dimensions (we will explain what this means to Section 7.4)?

In particular, we want to understand representations of $\mathfrak{p o}^{1 \mid N}$ satisfying some physics restrictions (most importantly, having the supersymmetry generators send bosons to only fermions, and vice versa; this kind of "swapping symmetry" is what supersymmetry tries to study). Understanding all such representations seems intractable, so we restrict our attention to representations where the supersymmetry generators act as permutations (up to a scalar) and also possibly the Hamiltonian $H=i \partial_{t}$ on the basis fields: we require that for any boson $\phi$ and any $Q_{I}$,

$$
Q_{I} \phi= \pm(-i H)^{s} \psi= \pm\left(\partial_{t}\right)^{s} \psi
$$

where $s \in\{0,1\}$, the sign, and the fermion $\psi$ depends on $\phi$ and $I$. We enforce a similar requirement

$$
Q_{I} \psi= \pm i(-i H)^{s} \phi= \pm i\left(\partial_{t}\right)^{s} \phi
$$

for fermions. We call the representations corresponding to these types of actions adinkraic representations. For each of these representations, we associate an adinkra. We now form a correspondence between our definition of adinkras in Section 2.1 and our definition for adinkraic representations. 


\begin{tabular}{c|c} 
adinkras & representations \\
\hline vertex bipartition & bosonic/fermionic bipartition \\
colored edges and $q_{I}$ & action of $Q_{I}$ without the sign or powers of $(-i H)$ \\
dashing / sign in $\bar{q}_{I}$ & sign in $Q_{I}$ \\
change of rank by $q_{I}$ and $\bar{q}_{I}$ & powers of $(-i H)$ in $Q_{I}$ \\
ranking & partition of fields by engineering dimension
\end{tabular}

To summarize,

An adinkra encodes a representation of $\mathfrak{p o}^{1 \mid N}$. An adinkraic representation is a representation of $\mathfrak{p o}^{1 \mid N}$ that can be encoded into an adinkra.

So instead of attacking Question 3.1 head-on, we focus on the following problem instead:

Question 3.2. What are all the adinkraic representations of $\mathfrak{p o}^{1 \mid N}$ ?

The set of adinkraic representations is already rich enough to contain representations of interest. When the poset structure of our adinkra $A$ is a boolean lattice, we get the classical notion of superfield introduced in [23] by Salam and Strathdee. When the poset of $A$ is a height-2 poset (in which case we say that $A$ is a valise), we get [9]'s Clifford supermultiplet. By direct sums, tensors, and other operations familiar to the Lie algebras setting, it is possible to construct many more representations, a technique that has been extended to higher dimensions in [18.

\section{The Classification of MUltichromotopologies AND CHROMOTOPOLOGIES}

Compared to the relevant sections of the original literature, our approach is more general and compact, though we owe most ideas to the original work. We now classify multichromotopologies, chromotopologies, and adinkraizable chromotopologies; we also note the pleasant connections with codes and Clifford algebras.

4.1. Graph quotients and codes. In this section, we recover the main result (Theorem 4.5) classifying adinkraizable chromotopologies from the existing literature. However, our more general approach (using multigraphs) gives the benefit of easily obtaining classification theorems of multichromotopologies and chromotopologies that are very analogous in flavor.

We now give a quick review of codes (there are many references, including [19]). An $n$-codeword is a vector in $\mathbf{Z}_{2}^{n}$, which we usually write as $b_{1} b_{2} \cdots b_{n}, b_{i} \in \mathbf{Z}_{2}$. We distinguish two $n$-codewords $\overrightarrow{1_{n}}=11 \ldots 1$ and $\overrightarrow{0_{n}}=00 \ldots 0$, and when $n$ is clear from context we suppress the subscript $n$. The number of 1's in a codeword $v$ is called the weight of the string, which we denote by $\operatorname{wt}(v)$. We use $\bar{v}$ to denote the bitwise complement of $v$, which reverses 0's and 1's. For example, $\overline{00101}=11010$. An $(n, k)$-linear binary code (for this paper, we will not talk about any other kind of codes, so we will just say code for short) is a $k$-dimensional $\mathbf{Z}_{2}$-subspace of codewords. A code is even if all its codewords have weight divisible by 2 and doubly-even if all its codewords have weight divisible by 4 .

Consider the $n$-cubical chromotopology $I_{c}^{n}$. For any linear code $L \subset \mathbf{Z}_{2}^{n}$, the quotient $\mathbf{Z}_{2}^{n} / L$ is a $\mathbf{Z}_{2}$-subspace. Using this, we define the map $p_{L}$, which sends $I_{c}^{n}$ 
to the following multichromotopology, which we call the graph quotient (or quotient for short) $I_{c}^{n} / L$ :

- Let the vertices of $I_{c}^{n} / L$ be labeled by the equivalence classes of $\mathbf{Z}_{2}^{n} / L$ and define $p_{L}(v)$ to be the image of $v$ under the quotient $\mathbf{Z}_{2}^{n} / L$. When $L$ is an $(n, k)$-code, the preimage over every vertex in $I_{c}^{n} / L$ contains $2^{k}$ vertices, so $I_{c}^{n} / L$ has $2^{n-k}$ vertices.

- Let there be an edge $p_{L}(v, w)$ in $I_{c}^{n} / L$ with color $i$ between $p_{L}(v)$ and $p_{L}(w)$ in $I^{n} / L$ if there is at least one edge with color $i$ of the form $\left(v^{\prime}, w^{\prime}\right)$ in $\mathbf{Z}_{2}^{n}$, with $v^{\prime} \in p_{L}^{-1}(v)$ and $w^{\prime} \in p_{L}^{-1}(w)$.

Every vertex in $I_{c}^{n} / L$ still has degree $n$ (counting possible multiplicity) and the commutivity condition on the $q_{i}$ 's is unchanged under a quotient, so $I_{c}^{n} / L$ is indeed a multichromotopology. Denote its underlying multigraph by $I^{n} / L$. We now prove some properties of the quotient.

Proposition 4.1. The following hold for $A=I^{n} / L$ :

(1) A has a loop if and only if $L$ contains a codeword of weight 1 ; A has a double-edge if and only if $L$ contains a codeword of weight 2 . Thus, $A$ is a simple graph if and only if $L$ contains only codewords of weight 3 or greater.

(2) $A$ can be ranked if and only if $A$ is bipartite, which is true if and only if $L$ is an even code.

Proof. (1) Suppose $A$ has a loop. This means some edge $(v, w)$ in $I_{c}^{n}$ has both endpoints $v$ and $w$ mapped to the same vertex in the quotient. Equivalently, $(v-w) \in L$. However, $v$ and $w$ differ by a codeword of weight 1 . Suppose $A$ has a double-edge $(v, w)$ with colors $i$ and $j$. Since $q_{1}\left(q_{2}(v)\right)=v$ in $A=I_{c}^{n} / L$, for some $v^{\prime} \in p_{L}^{-1}(v)$, we must have in $I_{c}^{n}$ that $q_{1}\left(q_{2}\left(v^{\prime}\right)\right)-v^{\prime}$ is in $L$. But this is a weight 2 codeword with support in $i$ and $j$. The logic is reversible in both of these situations.

(2) Suppose $A$ were not bipartite; then $A$ has some odd cycle. One of the preimages of this cycle in $I_{c}^{n}$ is a path of odd length from some $v$ to some $w$ that both map to the same vertex under the quotient (i.e. $v-w \in L$ ). Since each edge changes the weight of the vertex by $1(\bmod 2), v-w$ must have an odd weight. Since $v-w \in L, L$ cannot be an even code. For the other direction, note that if $L$ were an even code, such odd cycles cannot occur.

Recall that any bipartite graph can be ranked by making a valise. If $A$ can be ranked via a rank function $h$, the sets $\{v \in V(A) \mid h(v) \cong 0$ $(\bmod 2)\}$ and $\{v \in V(A) \mid h(v) \cong 1(\bmod 2)\}$ must be a bipartition of $A$ because all the edges in $A$ change parity of $h$.

The most difficult condition to classify is being well-dashed, which is intricately connected with Clifford algebras. See Appendix A for a review of Clifford algebras and some related results we will need. Let an almost doubly-even code be a code where all codewords in the code have weight 0 or $1(\bmod 4)$. The following result extends the ideas used in proving [9, Theorem 4.4].

Proposition 4.2. The multichromotopology $A=I_{c}^{n} / L$ can be well-dashed if and only if $L$ is an almost doubly-even code.

Proof. Given a codeword $v=v_{1} v_{2} \cdots v_{n}$, define $\bar{q}_{v}$ to be the map $\bar{q}_{n}^{v_{n}} \cdots \bar{q}_{1}^{v_{1}}$. 
Suppose we have an odd dashing. Let $v$ and $w$ be codewords in $L$. Both $\bar{q}_{v}$ and $\bar{q}_{w}$ take any vertex to itself in $\mathbf{R}[V(A)]$ with possibly a negative sign, since the $\bar{q}_{i}$ are basically the $q_{i}$ with possibly a sign, and following a sequence of $q_{i}$ corresponding to a codeword is a closed loop in $I_{c}^{n} / L$. This means $\bar{q}_{v}$ and $\bar{q}_{w}$ must commute; furthermore, $\bar{q}_{v}^{2}$ must be the identity map for any $v \in L$. By Lemma A.2, this is exactly the condition required for $L$ being an almost-doubly even code.

Now, suppose $L$ were an almost doubly-even code. Then by Proposition A.3. we can find a sign function $s$ such that $\{s(v) \operatorname{clif}(v) \mid v \in L\}$ forms a subgroup SMon $_{L} \subset$ SMon, the signed monomials of $\mathbf{C l}(n)$. This gives a well-defined action of SMon on $\mathbf{S M o n}_{\mathbf{S}} \mathbf{S M o n}_{L}$ via left multiplication while possibly introducing signs. The cosets of $\mathbf{S M o n}_{L}$ under SMon naturally correspond to $V(A)$, so we can define $\bar{q}_{i}(v)$ to introduce the same sign as $\gamma_{i}$ on $\operatorname{clif}(v) \in \mathbf{S M o n} / \mathbf{S M o n}_{L}$. Since we have a Clifford algebra action, we get the desired anticommutation relations between $\bar{q}_{i}$ and thus an odd dashing.

Quotients of $I_{c}^{n}$ are multichromotopologies. Surprisingly, the converse is also true, which gives us our main classification:

Theorem 4.3. Multichromotopologies are exactly quotients $I_{c}^{n} / L$ for some code $L$.

Proof. Take a multichromotopology $A$. Consider the abelian group $G$ acting on $V(A)$ generated by the $q_{i}$. The elements of $G$ can be written as $g=q_{1}^{s_{1}} q_{2}^{s_{2}} \cdots q_{n}^{s_{n}}$, where $s_{i} \in \mathbf{Z}_{2}$ for all $i$. Consider the isomorphism $\phi: G \rightarrow L$ which sends such a $g$ to the $n$-codewords $s_{1} s_{2} \cdots s_{n} \in \mathbf{Z}_{2}^{n}$. Take any vertex $v_{0} \in V(A)$ and consider its stabilizer group $H$ under $G . \phi(H)$ is a subspace of $\mathbf{Z}_{2}^{n}$ and thus must be some code $L$. Any vertex $v$ is equal to $g\left(v_{0}\right)$ for some $g \in G$, so we may label $v$ with the coset $\phi(g)+L$. It is easy to check that the resulting multichromotopology is exactly the one produced by the quotient $I_{c}^{n} / L$.

Combining Proposition 4.1 and Theorem 4.3 immediately gives the classification of all chromotopologies and adinkraizable chromotopologies:

Theorem 4.4. Chromotopologies are exactly quotients $I_{c}^{n} / L$, where $L$ is an even code with no codeword of weight 2 .

Theorem 4.5 (DFGHILM, basically [9, Theorem 4.4]). Adinkraizable chromotopologies are exactly quotients $I_{c}^{n} / L$, where $L$ is a doubly-even code.

Thanks to Theorem 4.3, we can assume the following:

From now on, any multichromotopology (including chromotopologies) $A$ we discuss comes from some $(n, k)$-code $L(A)=L$. If $L$ is an $(n, k)$-code, we say that the corresponding $A$ is an $(n, k)$-multichromotopology (or chromotopology).

An $(n, 0)$-multichromotopology must be the $n$-cubical chromotopology corresponding to the trivial code $\{\overrightarrow{0}\}$. The smallest non-cubical adinkraizable chromotopology, shown in Figure [5, is the quotient $I_{c}^{4} / L$ for $L=\{0000,1111\}$, the smallest non-trivial doubly-even code. Its underlying graph is the complete bipartite graph $K_{4,4}$.

While the problem of classifying adinkraizable chromotopologies reduces to that of classifying doubly-even linear codes, the theory of these codes is very rich and non-trivial. Computationally, 22] contains the current status of the classification 

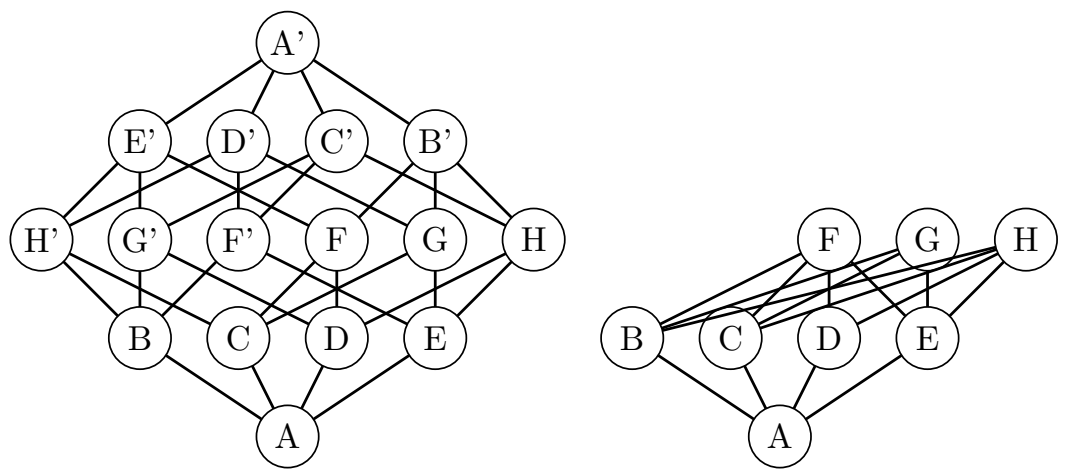

Figure 5. The graphs $I^{4}$ and $I^{4} /\{0000,1111\}$. Labels with the same letter are sent to the same vertex.

through an exhaustive search. We invite the reader to explore the other connections between adinkras and coding theory (for example, the irreducible adinkraic representations correspond to the self-dual codes) from the original sources, such as 9 .

Finally, we remark that studying well-dashed chromotopologies is basically equivalent to studying Clifford algebras. Some of this intuition is suggested by the proof of Proposition 4.2. We discuss this further in Section 7.3 .

4.2. A homological view. From the theorems in Section 4.1, we see that the $n$-cubical chromotopologies $I_{c}^{n}$ look like universal covers. We make this intuition rigorous in this section. We appeal to only basic techniques in homological algebra (any standard introduction, such as [17, is more than sufficient), but having another point of view may greatly enrich this new subject, as we show in Section 5 Happily, we have learned that there is some independent work in preparation ([5]) using similar homological techniques.

We work over $\mathbf{Z}_{2}$. Construct the following 2-dimensional complex $X(A)$ from a chromotopology $A$. Let $C_{0}$ be formal sums of elements of $V(A)$ and $C_{1}$ be formal sums of elements of $E(A)$. For each 2-colored 4-cycle $C$ of $A$, create a 2-cell with $C$ as its boundary as a generator in $C_{2}$. The boundary maps $\left\{d_{i}: C_{i} \rightarrow C_{i-1}\right\}$ are the natural choices (we do not worry about orientations since we are using $\mathbf{Z}_{2}$ ), giving homology groups $H_{i}=H_{i}(X(A))$. The most important observation about our complex $X(A)$ is the following, which we return to in Section 5.5.

Proposition 4.6. Let $A$ be an $(n, k)$-adinkraizable chromotopology with $L(A)=L$. Then $X(A)=X\left(I_{c}^{n}\right) / L$ as a quotient complex, where $L$ acts freely on $X\left(I_{c}^{n}\right)$. We have that $X\left(I_{c}^{n}\right)$ is a simply-connected covering space of $X(A)$, with $L$ the group of deck transformations.

Proof. The fact that $X(A)$ is a quotient complex is already evident from the construction of the graph quotient, since we have restricted to simple graphs (recall that adinkraizable chromotopologies have simple graphs). A quick way to see that $X\left(I_{c}^{n}\right)$ is simply-connected is note that $X\left(I_{c}^{n}\right)$ is the 2-skeleton of the $n$-dimensional (solid) hypercube $D^{n}$. Thus, $X\left(I^{n}\right)$ and $D$ must have matching $H_{1}$ and $\pi_{1}$, but $D$ is obviously simply-connected. 
4.3. Adinkra decomposition. Finally, we introduce a notion designed to reduce the complexity of chromotopologies. Say that a color $i$ decomposes a chromotopology $A$ if removing all edges of color $i$ splits $A$ into 2 separate connected components. This is equivalent to the underlying graph of $A$ being the cartesian product of a single edge and another graph. Our definition was inspired by observations in [9], where certain adinkras were called 1-decomposable. As Greg Landweber pointed out to us, the concept corresponding to decomposition in coding theory is punctured codes (see 19]).

Lemma 4.7. The color $i$ decomposes the chromotopology $A$ if and only if for all $d \in L(A)$, the $i$-th bit of $d$ is 0 .

Proof. This is a very straightforward verification. We leave the proof as an exercise to the reader.

Corollary 4.8. Every color in $[n]$ decomposes $I_{c}^{n}$.

In the situation where Lemma 4.7 holds, we say that $i$ decomposes $A$ into $A_{0}$ and $A_{1}$, or $A=A_{0} \amalg_{i} A_{1}$, if removing all edges with color $i$ creates two disjoint chromotopologies $A_{0}$ and $A_{1}$, which are labeled and colored in a natural fashion, equipped with an inclusion inc on their vertices that map into $V(A)$. Formally:

- $V(A)$ can be partitioned into two sets $V(A \mid 0)$ and $V(A \mid 1)$, where vertices in $V(A \mid 0)$ have 0 in the $i$-th bit (by Lemma 4.7, this is a well-defined notion) and vertices in $V(A \mid 1)$ have 1 in the $i$-th bit. Furthermore, all edges between $V(A \mid 0)$ and $V(A \mid 1)$ are of color $i$.

- Define $A_{0}$ to be isomorphic to the edge-colored graph induced by vertices in $V(A \mid 0)$, where any codeword $v=\left(b_{1} b_{2} \cdots b_{n}\right)$ in the vertex label class of $v^{\prime} \in$ $V(A \mid 0)$ is sent to the $(n-1)$-codeword $\left(b_{1} b_{2} \cdots \widehat{b_{i}} \cdots b_{n}\right)$, where we remove the bit $b_{i}$. Color the edges analogously with colors in $\{1,2, \ldots, \hat{i}, \ldots, n\}$. Define $A_{1}$ in the same way with $V(A \mid 1)$ instead of $V(A \mid 0)$.

- Define the maps inc $\left(b_{1} b_{2} \ldots b_{n-1}, j \rightarrow i\right)=b_{1} \ldots b_{i-1} j b_{i} \ldots b_{n-1}$, which inserts $j$ into the $i$-th place of an $(n-1)$-codeword to create an $n$-codeword. If $A=A_{0} \amalg_{i} A_{1}$, let $\operatorname{inc}(v)$ send a vertex $v \in A_{j}$ to $\operatorname{inc}(v, j \rightarrow i)$ for $j \in\{0,1\}$. Lemma 4.7 gives that the union of the image of $V\left(A_{0}\right)$ and $V\left(A_{1}\right)$ under inc is exactly $V(A)$.

Proposition 4.9. Let $A=A_{0} \amalg_{i} A_{1}$, where $A$ is an $(n, k)$-chromotopology. Then $A_{0}$ and $A_{1}$ are $(n-1, k)$ chromotopologies, isomorphic as graphs.

Proof. The image of $q_{i}$ on $V\left(A_{0}\right)$ is exactly $V\left(A_{1}\right)$ and $q_{i}$ is an involution, so we have a bijection between the vertices. If $q_{j}\left(v_{1}\right)=v_{2}$ in $A_{0}$, the 4 -cycle condition on $\left(v_{1}, q_{i}\left(v_{1}\right), q_{i}\left(v_{2}\right), v_{2}\right)$ gives that $\left(q_{i}\left(v_{1}\right), q_{i}\left(v_{2}\right)\right)$ is also an edge of color $j$ in $A_{1}$, so the bijection between the vertices extends to a bijection between $A_{0}$ and $A_{1}$ as edge-colored graphs, and thus chromotopologies. Each of these chromotopologies has $2^{n-1}$ vertices and is $(n-1)$-regular, so by Proposition 4.1 they must be $(n-1, k)$ chromotopologies.

\section{DASHING}

Given a chromotopology $A$, define $o(A)$ to be the set of odd dashings of $A$. Thus, adinkraizable chromotopologies are exactly those $A$ with $|o(A)|>0$.

Question 5.1. What are the enumerative and algebraic properties of $o(A)$ ? 

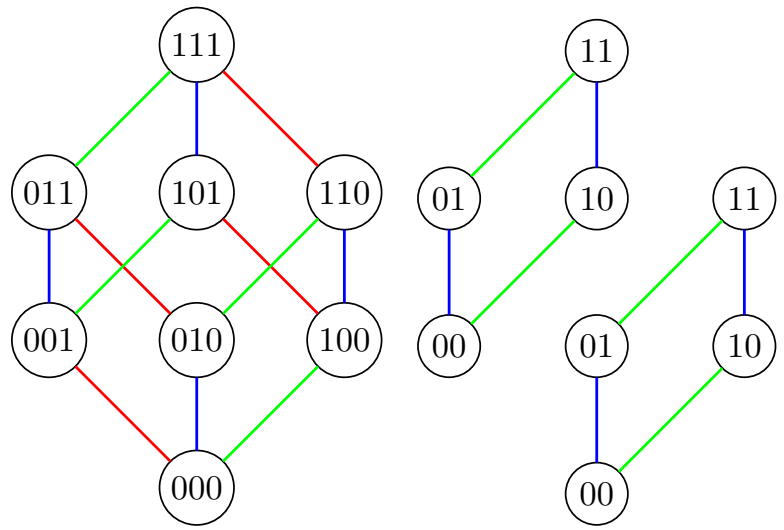

Figure 6 . The color 3 decomposes a ranked chromotopology $A$ (with chromotopology $I_{c}^{3}$ ) as $A=A_{0} \amalg_{3} A_{1}$. Each $A_{i}$ has chromotopology $I_{c}^{2}$.

In this section, we first generalize the concept of odd dashings to other graphs using the homological language we introduced in Section 4.2. We make some observations of this generalized setting from other parts of mathematics. Then, we return to the enumeration of dashings of adinkraizable chromotopologies. We introduce the concept of even dashings and relate them to odd dashings, showing that not only do the even dashings form a more convenient model for calculations, there is a bijection between the two types of dashings. We will then count the number of (odd or even) dashings of $I_{c}^{n}$ via a cute application of linear algebra. Happily, we are then able to generalize our formula to all chromotopologies with a homological algebra computation.

5.1. Generalizations and sightings of odd dashings. In Section 4.2, we created a (cubical) complex $X(A)$ from a chromotopology $A$ by using $A$ as the 1 skeleton and then attaching 2-cells for every 2-colored 4-cycle. One interpretation of this process is that we are distinguishing certain cycles (whose parities we care about when we dash $A$ ) and marking them via 2-cells.

Thus, an obvious generalization of odd dashings is the following: given a graph $A$ and a set of distinguished cycles $C$ in $A$, call a dashing $d: E(A) \rightarrow \mathbf{Z}_{2}$ an odd dashing of $(A, C)$ if for all cycles $c \in C$, the sum of $d(e)$ for $e$ in $c$ is odd. We can construct a 2-dimensional cell complex $X(A, C)$ by attaching 2-cells for every $c \in C$. When $A=I_{c}^{n} / L$ and $C$ is the set of 2-colored 4-cycles in $A$, we recover our original definitions.

The benefit of this generalization is that the odd dashings have a natural cohomological interpretation: associate a dashing of $(A, C)$ with an element of $H^{1}(X(A, C)$, $\mathbf{Z}_{2}$ ) in the natural way by sending each edge $e$ to $d(e)$. Then the distinguished element in $H^{2}(X(A, C))$ obtained by sending each 2-cell to 1 vanishes in cohomology if and only if there is an odd dashing. This suggests that the homological approach may make some proofs easier, as we will exhibit in Section 5.5. (As all our calculations are done in homology, we do not directly use the cohomological observation we just made in this paper. However, we would like to note that it plays a critical role in [5].) 
Remark 5.2. We want to remark that not only do these generalized odd dashings come up naturally in the study of adinkras, they have appeared elsewhere in mathematics under different disguises. A frivolous example is that making the signs of a total differential consistent in a double complex requires changing signs in the grid graph of differential maps such that every square has an odd number of sign changes (see, for example, [3]). A more sophisticated example is in [2, Lemma 10.4], where the authors needed to exhibit an odd dashing on a poset structure of the Weyl group of a Lie algebra $\mathfrak{g}$ in the construction of a $\mathfrak{g}$-module resolution. A uniform study of these occurrences would be very interesting. For the sake of length, we limit our study in this paper to adrinkraizable chromotopologies.

5.2. Even dashings. Let an even dashing be a way to dash $E(A)$ such that every 2 -colored 4-cycle contains an even number of dashed edges, and let $e(A)$ be the set of even dashings. We have the following nice fact:

Lemma 5.3. For any adinkraizable chromotopology $A$, we have $|o(A)|=|e(A)|$.

Proof. Let $l=|E(A)|$. We may consider a dashing of $A$ as a vector in $\mathbf{Z}_{2}^{l}$, where each coordinate corresponds to an edge and is assigned 1 for a dashed edge and 0 for a solid edge. There is an obvious way to add two dashings (i.e. addition in $\mathbf{Z}_{2}^{l}$ ) and there is a zero vector $d_{0}$ (all edges solid), so the family of all dashings (with no restrictions) forms a vector space $V_{\text {free }}(n)$ of dimension $l$.

Observe that $e(A)$ is a subspace of $V_{\text {free }}(n)$. To see this, we can directly check that adding two even dashings preserves the even parity of each 2-colored 4-cycle and that $d_{0}$ is an even dashing. Alternatively, we can note that the restriction of a dashing $d$ having a particular cycle with an even number of dashes just means the inner product of $d$ and some vector with four 1's as support is zero, so such dashings are exactly the intersection of $V_{\text {free }}(n)$ and a set of hyperplanes, which is a subspace.

Unlike the even dashings $e(A)$, the odd dashings $o(A)$ do not form a vector space; in particular, they do not include $d_{0}$. However, adding an even dashing to an odd dashing gives an odd dashing, and the difference between any two odd dashings gives an even dashing. Thus, $o(A)$ is a coset in $V_{\text {free }}(n)$ of $e(A)$ and must then have the same cardinality as $e(A)$ given that at least one odd dashing exists. Since $A$ is adinkraizable by definition, we are done.

The proof of Lemma 5.3 shows that the odd dashings form a torsor for the even dashings, which is easier to work with. We know from the results of Section 4.1 that certain quotients of $I_{c}^{n}$ are adinkraizable, including $I_{c}^{n}$ itself. But constructing an odd dashing for even $I_{c}^{n}$ is not obvious. Even dashings of $I_{c}^{n}$, however, are easy to construct (for example, just make all edges solid or make all edges dashed).

5.3. Decompositions and dashing $I_{c}^{n}$. We start by looking at the $n$-cubical chromotopology $I_{c}^{n}$. The main simplification here is that dashings behave extremely well under decompositions.

Lemma 5.4. If $A$ has $l$ edges colored $i$ and $A=A_{0} \amalg_{i} A_{1}$, then each even (resp. odd) dashing of the induced graph of $A_{0}$ and each of the $2^{l}$ choices of dashing the $i$-colored edges extends to exactly one even (resp. odd) dashing of $A$.

Proof. Without loss of generality, we can take $i=1$, so $A_{0}$ contains equivalence classes of codewords with first bit 0 and $A_{1}$ contains those with first bit 1 . 
After an even dashing of $A_{0}$ and an arbitrary dashing of the $i$-colored edges, note the remaining 2-colored 4-cycles are of exactly two types:

(1) the 4-cycles in $A_{1}$;

(2) the 4-cycles of the form $(u, v, w, x)$, where $(u, v)$ is in $A_{0},(w, x)$ is in $A_{1}$, and $(v, w)$ and $(x, u)$ are colored $i$.

Note that in all the cycles $(u, v, w, x)$ of the second type, $(w, x)$ is the only one we have not selected. Thus, there is exactly one choice for each of those edges to satisfy the even parity condition. Since there is exactly one such cycle for every edge in $A_{1}$, this selects a dashing for all the remaining edges, and the only thing we have to check is that the 4-cycles of the first type, the ones entirely in $A_{1}$, are evenly dashed.

Now, a 4-cycle of this type is of form $\left(1 a_{1}, 1 a_{2}, 1 a_{3}, 1 a_{4}\right)$, which is a face of a 3 cube with vertices $\left(0 a_{1}, 0 a_{2}, 0 a_{3}, 0 a_{4}, 1 a_{1}, 1 a_{2}, 1 a_{3}, 1 a_{4}\right)$. There are 5 other 4 -cycles in this hypercube which have all been evenly dashed (the $0 a_{i}$ vertices form a cycle in $A_{0}$ and the other 4 cycles are evenly dashed by our previous paragraph). Thus, we have that:

$$
\begin{aligned}
& d\left(0 a_{1}, 0 a_{2}\right)+d\left(0 a_{2}, 0 a_{3}\right)+d\left(0 a_{3}, 0 a_{4}\right)+d\left(0 a_{4}, 0 a_{1}\right)=0 \\
& d\left(0 a_{1}, 0 a_{2}\right)+d\left(0 a_{2}, 1 a_{2}\right)+d\left(1 a_{2}, 1 a_{1}\right)+d\left(1 a_{1}, 0 a_{1}\right)=0 \\
& d\left(0 a_{2}, 0 a_{3}\right)+d\left(0 a_{3}, 1 a_{3}\right)+d\left(1 a_{3}, 1 a_{2}\right)+d\left(1 a_{2}, 0 a_{2}\right)=0 \\
& d\left(0 a_{3}, 0 a_{4}\right)+d\left(0 a_{4}, 1 a_{4}\right)+d\left(1 a_{4}, 1 a_{3}\right)+d\left(1 a_{3}, 0 a_{3}\right)=0 \\
& d\left(0 a_{4}, 0 a_{1}\right)+d\left(0 a_{1}, 1 a_{1}\right)+d\left(1 a_{1}, 1 a_{4}\right)+d\left(1 a_{4}, 0 a_{4}\right)=0
\end{aligned}
$$

Adding these equations in $\mathbf{Z}_{2}$ gives:

$$
d\left(1 a_{1}, 1 a_{2}\right)+d\left(1 a_{2}, 1 a_{3}\right)+d\left(1 a_{3}, 1 a_{4}\right)+d\left(1 a_{4}, 1 a_{1}\right)=0 .
$$

Thus, we have constructed an even dashing. The analogous result for odd dashings follows if we replace the 0's on the right sides of the above equations by 1's.

Remark 5.5. This proof is easily seen to generalize to dashings of $(A \times I, C)$, where the cycles of $C$ occur in a "mirrored" fashion in the two halves of the graph, and all 4-cycles that use the edges corresponding to $I$ belong to $C$.

Proposition 5.6. The number of even (or odd) dashings of $I_{c}^{n}$ is

$$
\left|e\left(I_{c}^{n}\right)\right|=\left|o\left(I_{c}^{n}\right)\right|=2^{2^{n}-1} .
$$

Proof. A convenient property of hypercubes is that every 4-cycle is a 2-colored 4cycle. Thus, we get to just say "4-cycles" instead of "2-colored 4-cycles" in this proof.

We prove our result by induction. The base case is easy: for $n=1$ (a single edge), there are exactly 2 even dashings, since there is no 4-cycle. Suppose our result were true for every $k<n$. We will now show it is also true for $n$. Recall from Corollary 4.8 that every color decomposes $I_{c}^{n}$ into two smaller $I_{c}^{n-1}$ 's. Since we have $2^{n-1}$ edges with color 1 , by Lemma 5.4 we get the recurrence

$$
\left|e\left(I_{c}^{n}\right)\right|=2^{2^{n-1}}\left|e\left(I_{c}^{n-1}\right)\right| .
$$

With the initial case $\left|e\left(I_{c}^{1}\right)\right|=2$, we get $\left|e\left(I_{c}^{n}\right)\right|=2^{2^{n-1}+2^{n-2}+\cdots+1}=2^{2^{n}-1}$, as desired. The result for $\left|o\left(I_{c}^{n}\right)\right|$ is immediate by Lemma 5.3. 
Note that $\left|o\left(I_{c}^{n}\right)\right|=2^{2^{n}} / 2$. This suggests that, besides a single factor of 2 , each of the $2^{n}$ vertices gives exactly one "degree of freedom" for odd dashings. We will justify this hunch in the following discussion, in particular with Proposition 5.7.

5.4. Vertex switching. In [10], Douglas, Gates, and Wang examined dashings from a point of view inspired by Seidel's two-graphs (24]). Define the vertex switch at a vertex $v$ of a well-dashed chromotopology $A$ as the operation that produces the same $A$, except with all edges adjacent to $v$ flipped in parity (sending dashed edges to solid edges, and vice versa). It is routine to verify that a vertex switch preserves odd dashings (in fact, parity in all 4-cycles), so the odd dashings of $A$ can be split into orbits under all possible vertex switchings, which we will call the labeled switching classes (or LSCs) of $A$. We emphasize the adjective "labeled" because the term switching class in [10] refers to equivalence classes not only under vertex switchings, but also under different types of vertex permutations.
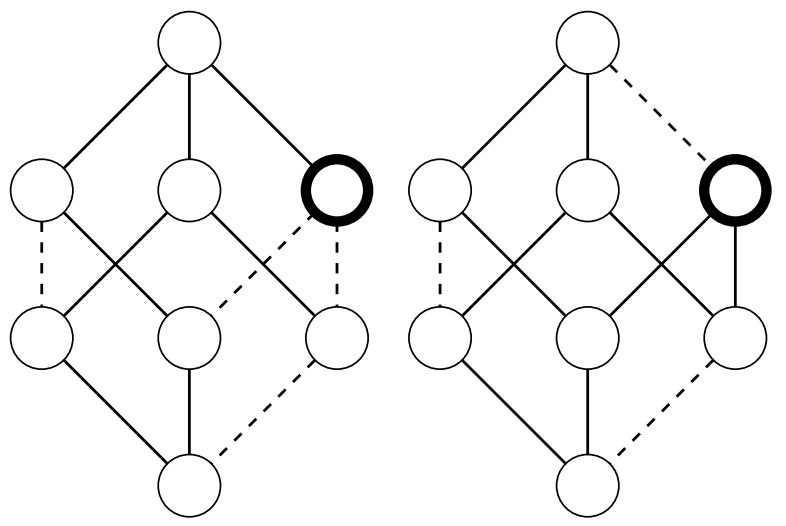

FIgURE 7 . Before and after a vertex switch at the outlined vertex.

In the representation theory interpretation of adinkras (see Section 3), a vertex switch corresponds to adding a negative sign in front of a component field, which gives an isomorphic representation. Thus, it is natural to think about equivalence classes under these transformations. The following computation will not only be useful to study switchings, but will also justify our hunch about the "degrees of freedom" from Proposition 5.6 .

Proposition 5.7. In an adinkraizable $(n, k)$-chromotopology $A$, there are exactly $2^{2^{n-k}-1}$ dashings in each LSC.

Proof. Vertex switches commute and each vertex switch is an order-2 operation, so they form a $\mathbf{Z}_{2}$-vector space, which we may index by subsets of the vertices. Consider a set of vertex switches that fix a dashing. Then, each edge must have its two vertices both switched or both non-switched. This decision can only be made consistently over all vertices if all vertices are switched or all vertices are non-switched. Thus, the $2^{n-k}$ sets of vertex switches generate a $\mathbf{Z}_{2}$-vector space of dimension $2^{n-k}-1$. This proves the result.

Corollary 5.8. The cubical chromotopology $I_{c}^{n}$ has exactly one labeled switching class. 
Proof. This is immediate from Proposition [5.7 and Proposition [5.6] with the substitution $k=0$. Alternatively, this is also evident from [10, Lemma 4.1].

5.5. A homological computation. Finally, we combine several ideas (even dashings, vertex switchings, and our cell complex interpretation of chromotopologies) to generalize Proposition 5.6

Proposition 5.9. Let $A$ be an adinkraizable $(n, k)$-chromotopology. Then there are $2^{k}$ LSCs in $A$.

Proof. First, vertex switchings preserve parity of all 4-cycles, so counting orbits of odd dashings (LSCs) under vertex switchings is equivalent to counting orbits of even dashings.

An even dashing can also be thought of as a formal sum of edges over $\mathbf{Z}_{2}$ (we dash an edge if the coefficient is 1 and do not otherwise), which is precisely a 1chain of $X(A)$ over $\mathbf{Z}_{2}$. Second, the even dashings are defined as dashings where all 2-colored 4-cycles have sum 0 . Since these 4-cycles, as elements of $C_{1}$, are exactly the boundaries of $C_{2}$, the even dashings are exactly the orthogonal complement of $\operatorname{Im}\left(d_{2}\right)$ inside of $C_{1}$ by the usual inner product. Thus, the even dashings have $\mathbf{Z}_{2}$-dimension:

$$
\begin{aligned}
\operatorname{dim}\left(\left(\operatorname{Im}\left(d_{2}\right)^{\perp}\right)\right. & =\operatorname{dim}\left(C_{1}\right)-\operatorname{dim}\left(\operatorname{Im}\left(d_{2}\right)\right) \\
& =\left(\operatorname{dim}\left(\operatorname{ker}\left(d_{1}\right)\right)+\operatorname{dim}\left(\operatorname{Im}\left(d_{1}\right)\right)\right)-\operatorname{dim}\left(\operatorname{Im}\left(d_{2}\right)\right) \\
& =\operatorname{dim}\left(H_{1}\right)+\operatorname{dim}\left(\operatorname{Im}\left(d_{1}\right)\right) \\
& =\operatorname{dim}\left(H_{1}\right)+\operatorname{dim}\left(C_{0}\right)-\operatorname{dim}\left(H_{0}\right) .
\end{aligned}
$$

However, note that $\operatorname{dim}\left(C_{0}\right)-\operatorname{dim}\left(H_{0}\right)=2^{n-k}-1$, which is exactly the dimension of the vector space of the vertex switchings for a particular LSC from Proposition 5.7. Since the product of the number of LSCs and the number of vertex switchings per LSC is the total number of even dashings, dividing the number of even dashings by $2^{2^{n-k}-1}$ gives that the dimension of switching classes is precisely $\operatorname{dim}\left(H_{1}\right)$.

By Proposition 4.6 and basic properties of universal covers and fundamental groups, $\pi_{1}(X(A))=L$, the quotient group, which in this case is the vector space $\mathbf{Z}_{2}^{k}$. Also, $H_{1}=\mathbf{Z}_{2}^{k}$ since $H_{1}$ is the abelianization of $\pi_{1}$. Thus, we have $2^{k}$ switching classes.

Propositions 5.9 and 5.7 immediately give:

Theorem 5.10. The number of even (or odd) dashings of an adinkraizable $(n, k)$ chromotopology A is

$$
|e(A)|=|o(A)|=2^{2^{n-k}+k-1} .
$$

A surprising but neat consequence of this result is that the number of dashings does not depend on the actual code $L(A)$, rather just on its dimension. This is very non-intuitive to see with elementary combinatorial methods.

\section{RANKING}

We now turn our study to rankings. Unlike dashing, most of our results on ranking do not depend on the chromotopology property at all and thus apply to all bipartite graphs. Thus, we can also think of this section as a general study of putting graded poset structures on arbitrary bipartite graphs. 
Fix a bipartite graph $A$. There will be an infinite number of maps $h: V(A) \rightarrow \mathbf{Z}$, but recall that each ranking is an equivalence class of these maps up to translation, so there are only a finite number of inequivalent rankings. In this section, when the context is clear, we will use rank functions $h$ interchangeably with their corresponding ranked graphs, so we can efficiently say "the ranking $A$ " instead of "the ranked graph $A$ " or "the sinks of $h$ " instead of "the sinks of $A$ when $A$ is equipped with the rank function $h$ ". We will also use rank functions interchangeably with their equivalence classes.

Call the set of all rankings of $A$ the rank family $R(A)$ of $A$. Figure 8 shows the rank family of $I^{2}$.
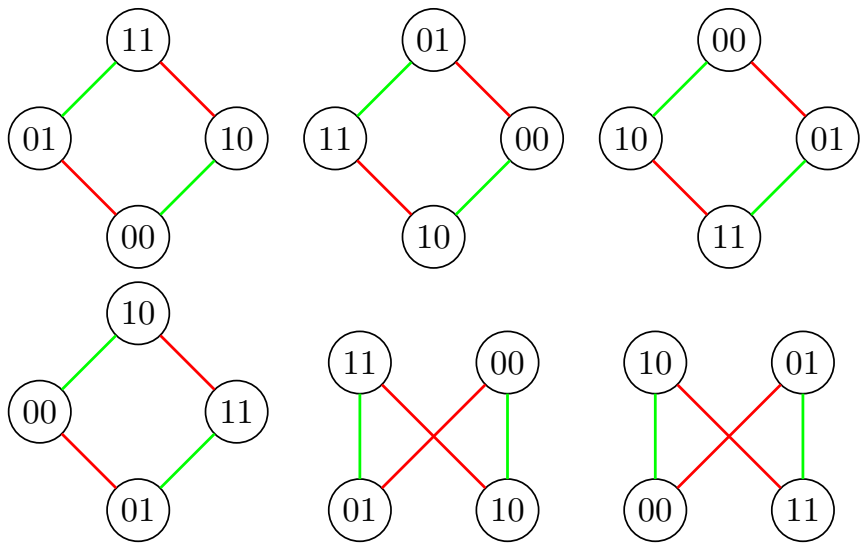

Figure 8 . The rank family of $I^{2}$.

Question 6.1. What are the enumerative and algebraic properties of $R(A)$ ?

After a short survey of the literature (mostly developed in 7]), we give some original results using the language of posets and lattices in Section 6.3. Finally, with the help of decomposition, we will computationally enumerate the possible rankings for $I_{c}^{n}$ with $n \leq 5$.

6.1. Hanging gardens. The main structural theorem for rankings is the following theorem. Let $D(v, w)$ be the graph distance between $v$ and $w$.

Theorem 6.2 (DFGHIL, [7, Theorem 4.1]). Fix a bipartite graph A. Let $S \subset V(A)$ and $h_{S}: S \rightarrow \mathbf{Z}$ satisfy the following properties:

(1) $h_{S}$ takes only odd values on bosons and only even values on fermions, or vice versa.

(2) For every distinct $s_{1}$ and $s_{2}$ in $S$, we have $D\left(s_{1}, s_{2}\right) \geq\left|h_{s}\left(s_{1}\right)-h_{s}\left(s_{2}\right)\right|$.

Then, there exists a unique ranking $h$ of $A$, such that $h$ agrees with $h_{S}$ on $S$ and $h$ 's set of sinks is exactly $S$. By symmetry, there also exists a unique ranking of $A$ whose set of sources is exactly $S$.

In other words, any ranking of $A$ is determined by a set of sinks (or sources) and the relative ranks of those sinks/sources. We can visualize such a choice as thus: pick some nodes as sinks and "pin" them at acceptable relative ranks, and let the 
other nodes naturally "hang" down. Thus, Theorem6.2 is also called the "Hanging Gardens" Theorem. Figure 9 shows an example. If we choose sources instead of sinks, we can imagine pinning down those nodes and having the other nodes "floating" up; the name "Floating Gardens" evokes an equally pleasant image.
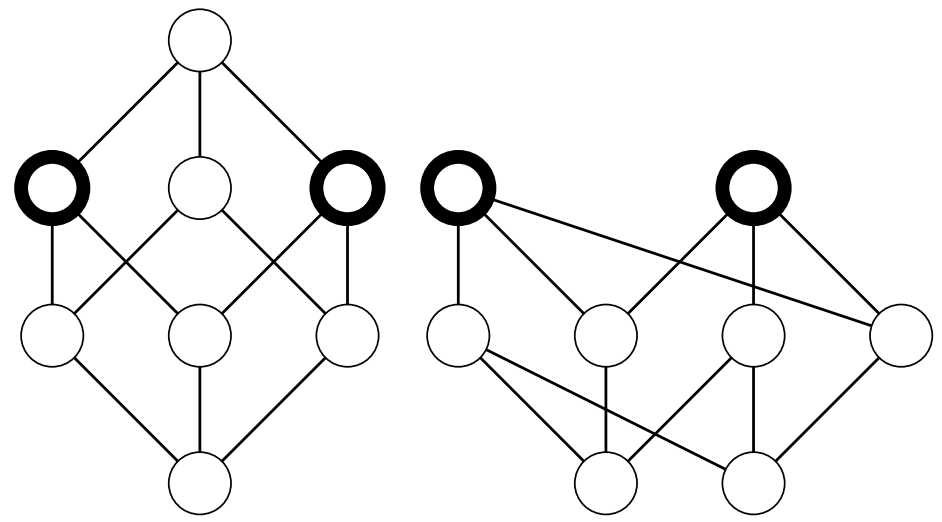

Figure 9. Left: $I^{3}$. Right: Hanging Gardens on $I^{3}$ applied to the two outlined vertices.

In particular, note that we can pick the set of sinks to contain only a single element, which defines a unique ranking. Thus, for any vertex $v$ of a bipartite graph $A$, by Theorem 6.2 we can get a ranking $A^{v}$ defined by its only having one sink $v$ (visually, $A^{v}$ "hangs" from its only sink $v$ ). We call $A^{v}$ the $v$-hooked ranking and all such rankings one-hooked. By symmetry, we can also define the $v$-anchored ranking $A_{v}$, which "floats" from its only source $v$. For example, Figure 3 is both the 111-hooked ranking $A^{111}$ and the 000-anchored ranking $A_{000}$ of $I^{3}$.

Now, we introduce two operators on $R(A)$. Given a ranking $B$ in $R(A)$ (with rank function $h$ ) and a sink $s$, we define $D_{s}$, the vertex lowering on $s$, to be the operation that sends $B$ to the ranking $h^{\prime}$ where everything is unchanged except $h^{\prime}(s)=h(s)-2$ (visually, we have "flipped" $s$ down two ranks and its edges with it). Observe that since $s$ was a sink, this operation retains the fact that all covering relations have rank difference 1 and thus we still get a ranking. We define $U_{s}$, the vertex raising on $s$, to be the analogous operation for $s$ a source. We call both of these operators vertex flipping operators.

Proposition 6.3 (DFGHIL, [7, Theorem 5.1, Corollary 5.2]). Let $A$ be a ranking. For any vertex $v$ :

(1) we can obtain $A^{v}$ from $A$ (or $A$ from $A_{v}$ ) via a sequence of vertex lowerings;

(2) we can obtain $A$ from $A^{v}$ (or $A_{v}$ from $A$ ) via a sequence of vertex raisings.

Furthermore, in any of these sequences we do not need to ever raise or lower $v$.

Proof. The main idea of the proof is again visually intuitive: starting with any $A$, "pin" $v$ to a fixed rank and let everything else fall down by gravity (slightly more formally: greedily make arbitrary vertex lowerings, except on $v$, until it is no longer possible). The result is easily seen to be $A^{v}$. The other claims follow by symmetry. 
Corollary 6.4. Any two rankings with the same graph $A$ can be obtained from each other via a sequence of vertex-raising or vertex-lowering operations.

Corollary 6.4 shows that there exists a connected graph $G$ with $V(G)=R(A)$ and $E(G)$ corresponding to vertex flips. In the literature (say [7]), $R(A)$, equipped with this graph structure, is called the main sequence.

6.2. The rank family poset. Consider a bipartite graph $A$. We know from the discussion in the previous section that its rank family has the structure of a graph. In this section, we show that it actually has much more structure. Our main goal will be to explicitly prove some observations made in [7, Section 8].

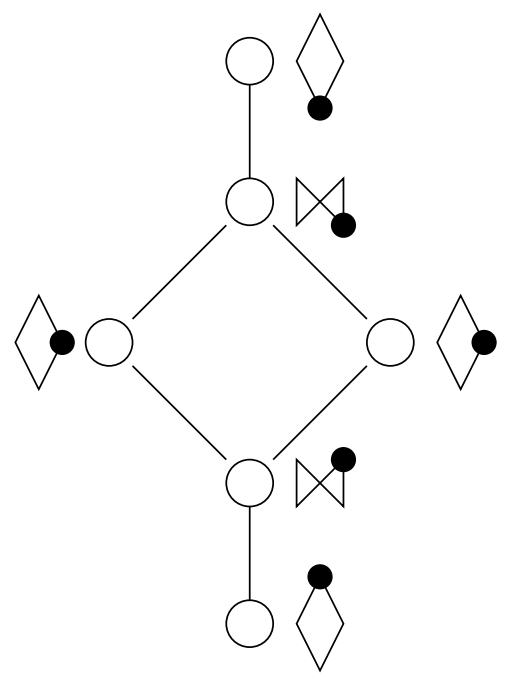

Figure 10. The rank family poset for $P_{v}\left(I^{2}\right)$, where next to each node is a corresponding ranking. The rankings are presented as miniature posets, with the black dots corresponding to $v$, the vertex at which we are not allowed to raise.

Theorem 6.5. For a bipartite graph $A$ and any vertex $v$ of $A$, there exists a poset $P_{v}(A)$ such that:

(1) $R(A)=V\left(P_{v}(A)\right)$;

(2) $P_{v}(A)$ is a symmetric ranked poset, with exactly one element in the top rank and exactly one element in the bottom rank;

(3) each covering relation in $P_{v}(A)$ corresponds to vertex-flipping on some vertex $w \neq v$.

Proof. Construct $P_{v}(A)$, as a ranked poset, in the following way: on the bottom rank 0 put $A^{v}$ as the unique element. Once we finish constructing rank $i$, from any element $B$ on rank $i$, perform a vertex-raising on all sources (except $v$ ) to obtain a set of rankings $S(B)$. Put the union of all $S(B)$ (as $B$ ranges through the elements on rank $i$ ) on rank $i+1$, adding covering relations $C>B$ if we obtained $C$ from $B$ via a vertex-raising. It is obvious from this construction that the covering relations in $P_{v}(A)$ are exactly the vertex flippings on vertices that are not $v$. 
We stop this process if all the elements of rank $i$ have no sources besides $v$ to raise. By Theorem 6.2, this is only possible for a single ranking, namely $A_{v}$. Thus, there is exactly one element in the top rank of $P_{v}(A)$ as well. By Proposition 6.3, we can get from $A^{v}$ to any element of $R(A)$ by vertex-raising only, without ever raising $v$. This means that every element of $R(A)$ has appeared exactly once in our construction.

Now, consider the map $\phi$ that takes a ranking $B$ of rank $k$ to the ranking $B^{\prime}$, in which any $v$ with rank $i$ in $B$ has rank $k-i$ in $B^{\prime}$. Since $\phi$ takes $A_{v}$ to $A^{v}$, and vice versa, the top and bottom ranks are symmetric. However, $\phi$ also switches, covering relations of vertex-raisings to vertex-lowerings. Thus, we can show that for every $i$ the $i$-th ranks and the $(k-i)$-th ranks are symmetric by induction on $i$. This makes $P_{v}(A)$ into a symmetric poset as desired.

For a ranking $B \in R(A)$, we now explicitly define $D_{s}(B)$ or $U_{s}(B)$ to 0 if the corresponding flip is not allowed. This allows us to consider $D_{s}$ and $U_{s}$ as operators $F[R(A)] \rightarrow F[R(A)]$, where $F$ is an arbitrary field and $F[R(A)]$ are formal sums of rankings over $F$. Define $U(A)$ to be the operator algebra generated by all $U_{s}$ with $s \in V(A)$.

Corollary 6.6. The image of $A^{v}$ under the action of the quotient $U(A) / U_{v}$ is $\mathbf{R}[R(A)]$.

Proof. This is immediate from the construction in Theorem 6.5, where we started with a single ranking $A^{v}$. Taking the image under vertex raisings is exactly the same as taking the image of the $U(A)$-action on $A^{v}$. Forbidding the vertex raising at $v$ is exactly the same as restricting this action to the quotient $U(A) / U_{v}$.

The authors of [7] noted that the rank family is reminiscent of a Verma module. Corollary 6.6 is an algebraic realization of this observation. The ranking $A^{v}$ takes the role of the lowest-weight vector. If we allowed vertex raisings at $v$, we would have obtained an infinite repeating family of rankings, as in Figure 11. When we strip the redundant rankings by forbidding $U_{v}$, we leave ourselves with a finite $R(A)$.

6.3. Revisiting the hanging gardens. In this section, we will put even more structure on $P_{v}(A)$ with the language of lattices. Again, any standard reference such as 25] would be sufficient.

We first construct an auxiliary poset $E_{v}(A)$, which we call the $v$-elevation poset of $A$ :

- let the vertices of $E_{v}(A)$ be ordered pairs $(w, h)$, where $w \in A, w \neq v$, and $h \in\{1,2, \ldots, D(w, v)\}$;

- whenever $D\left(w_{1}, w_{2}\right)=1$ and $D\left(w_{1}, v\right)+1=D\left(w_{2}, v\right)$, have $\left(w_{1}, h\right)$ cover $\left(w_{2}, h\right)$ and $\left(w_{2}, h+1\right)$ cover $\left(w_{1}, h\right)$.

Our main result of this section is the following theorem.

Theorem 6.7. The v-elevation poset and the v-rank family poset are related by

$$
P_{v}(A)=J\left(E_{v}(A)\right) .
$$

Thus, the rank family poset $P_{v}(A)$ is a finite distributive lattice. 

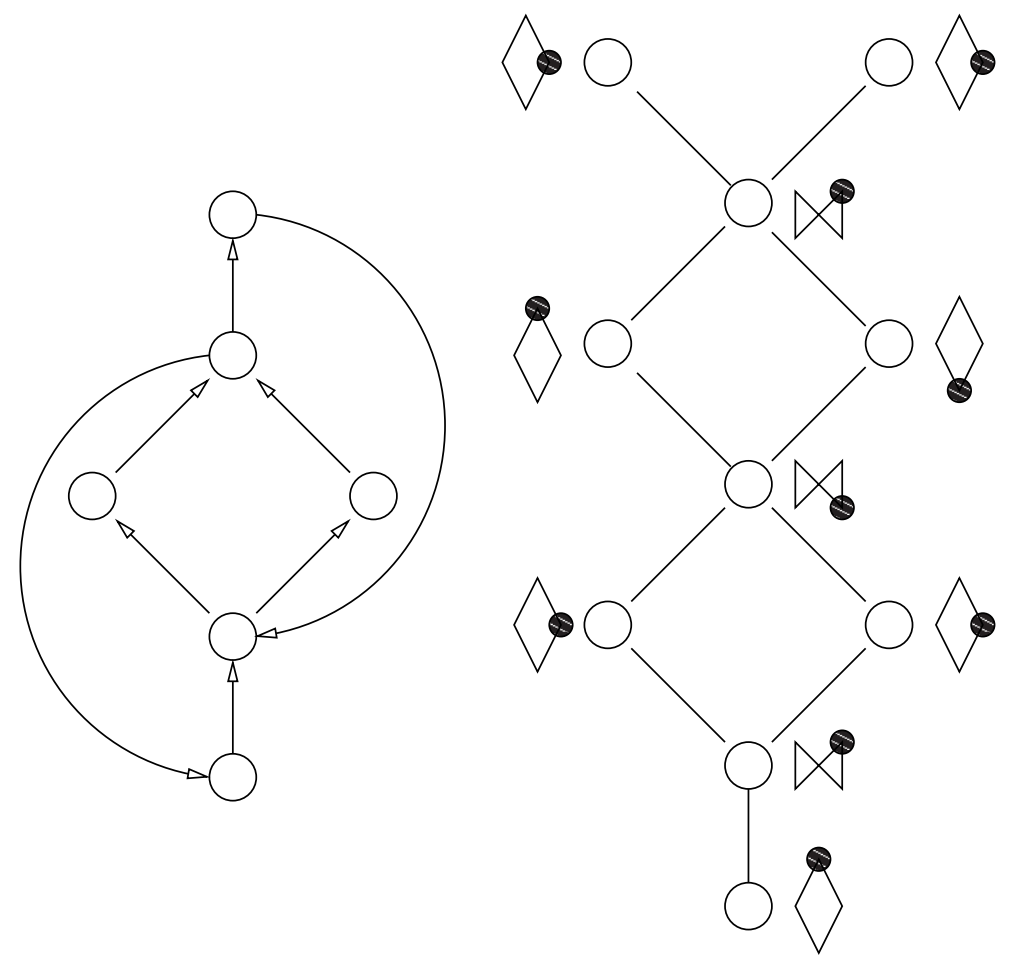

FiguRE 11. Left: if we were to allow vertex raising at $v$, we would no longer get a poset since we introduce cycles. Right: we can also think of this situation by an infinite poset leading upwards.

Proof. We show that there is a bijection between order ideals of $E_{v}(A)$ and elements of $P_{v}(A)$. The second claim in the theorem follows immediately from the Fundamental Theorem for Finite Distributive Lattices.

Each ideal $I$ of $E_{v}(A)$ gives exactly one ranking in $P_{v}(A)$, as follows: for every vertex $w \in A$, take the maximum $h \in \mathbf{Z}$ such that $(w, h) \in I$, taking $h=0$ if no $(w, h)$ appears in $I$. Now assign to $w$ the rank $2 h-D(w, v)$. In other words, $h$ indexes the elevation of $w$ by counting the number of total times we flip $w$ up from the initial state of the $v$-hooked ranking (which corresponds to the empty ideal), justifying the name elevation poset. It remains to check that this map is a bijection.

Take a ranking $A^{\prime}$ in $P_{v}(A)$. For any $w \in A^{\prime}$, we have $h(w)=2 h-D(w, v)$ for some $0 \leq h \leq D(w, v)$. Define $I \subset V\left(E_{v}(A)\right)$ to contain all $\left(w, h^{\prime}\right)$, possibly empty, with $h^{\prime} \leq h$. The property of $A^{\prime}$ being a ranking is equivalent to the condition that for every pair of neighbors $w_{1}$ and $w_{2}$ with $D\left(v, w_{2}\right)=D\left(v, w_{1}\right)+1$, we have $\left|h\left(w_{1}\right)-h\left(w_{2}\right)\right|=1$. However, this in turn is equivalent to the condition that the maximal $h_{1}$ and $h_{2}$ such that $\left(w_{1}, h_{1}\right)$ and $\left(w_{2}, h_{2}\right)$ appear (as before, define one of them to be 0 if no corresponding vertices exist in $I$ ) in $I$ satisfy either $h_{1}=h_{2}$ or $h_{1}=h_{2}+1$, which is exactly the requirement for $I$ to be an order ideal. Thus, our bijection is complete.

The proof of Theorem 6.7 gives another interpretation of Theorem 6.2. Consider the order ideals of $E_{v}(A)$. Each such order ideal corresponds to an antichain of 


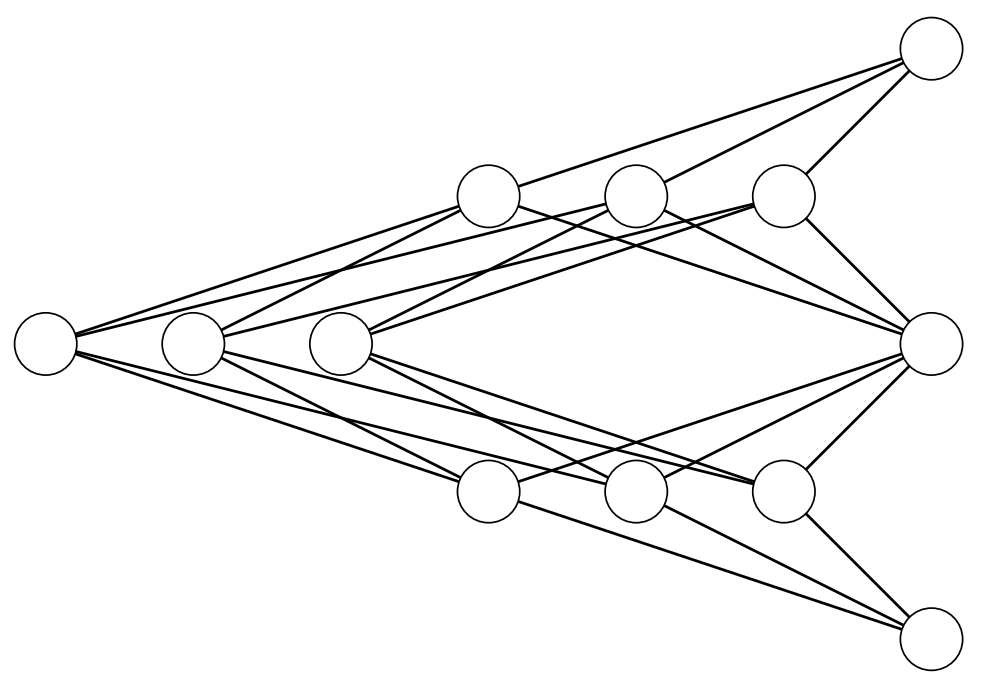

Figure 12. The elevation poset $E_{000}\left(I_{c}^{3}\right)$. Nodes $(w, h)$ on each vertical line have the same $w$-value. In order (left to right), the $w$-values are: 100, 010,001, 110,101,011, 111.

maximal elements, which is some collection of $\left(w_{i}, h\left(w_{i}\right)\right)$. It can be easily checked that in the corresponding element of $P_{v}(A)$, the $w_{i}$ are exactly the sinks, placed at rank $2 h_{i}-D\left(w_{i}, v\right)$.

Even though Theorem 6.7 gives us more structure on the rank family, it is very difficult in general to count the order ideals of an arbitrary poset. The typical cautionary tale is the case of the (extremely well-understood) Boolean algebra $B_{n}$, where the problem of counting the order ideals, known as Dedekind's Problem, has resisted a closed-form solution to this day, with answers computed up to only $n=8$ (see [28]).

6.4. Counting rankings of the cubical chromotopology. For a general bipartite graph $A$, counting the cardinality of $R(A)$ seems to be difficult, even when $A=I_{n}^{c}$. We perform an algorithmic attack with the help of decomposition.

Suppose $A=A_{0} \amalg_{i} A_{1}$. Now, let $z_{0}=\operatorname{inc}\left(\overrightarrow{0_{n-1}}, 0 \rightarrow i\right)$ and $z_{1}=\operatorname{inc}\left(\overrightarrow{0_{n-1}}, 1 \rightarrow i\right)$ be elements in $V(A)$. Since they are adjacent, their rank functions must differ by exactly 1 ; that is, $\left|h\left(z_{0}\right)-h\left(z_{1}\right)\right|=1$. We denote $A=A_{0} \nearrow_{i} A_{1}$ in the case $h\left(z_{1}\right)=h\left(z_{0}\right)+1$ and $A=A_{0} \searrow_{i} A_{1}$ otherwise. With this notation, we reinterpret Figure [6] as $A_{0} \nearrow_{3} A_{1}$, where we have $\nearrow$ because $000>001$ in $A$.

For any $A \in R\left(I_{c}^{n}\right)$, the color $n$ must decompose $A$ uniquely into $A_{0} \nearrow_{n} A_{1}$ or $A_{0} \searrow_{n} A_{1}$, where each of $A_{0}$ and $A_{1}$ is a ranking in $R\left(I_{c}^{n-1}\right)$. Thus, we can iterate over potential pairs of rankings $\left(A_{0}, A_{1}\right)$ and see if each of them could have come from some $A$. It suffices to check that each pair of vertices inc $(c, 0 \rightarrow n)$ and $\operatorname{inc}(c, 1 \rightarrow n)$, where $c \in \mathbf{Z}_{2}^{n-1}$, has rank functions differing by exactly 1 . However, this requires $2^{n-1}$ comparisons for each pair of ranking in $R\left(I^{n-1}\right)$. The following lemma cuts down the number of comparisons.

Lemma 6.8. For an $(n, k)$-ranking $A$ and $(n-1, k)$-rankings $A_{0}$ and $A_{1}$, we have $A=A_{0} \nearrow_{n} A_{1}$ if and only if the colors and vertex labelings of the three rankings are 
consistent and the following condition is satisfied: for each $c \in \mathbf{Z}_{2}^{n-1}$ and the pair of corresponding vertices $s_{0}=\operatorname{inc}(c, 0 \rightarrow n)$ and $s_{1}=\operatorname{inc}(c, 1 \rightarrow n)$ such that at least one of $s_{0}$ or $s_{1}$ is a sink (in $A_{0}$ or $A_{1}$, respectively), we have $\left|h\left(s_{0}\right)-h\left(s_{1}\right)\right|=1$.

Proof. These are clearly both necessary conditions. It obviously suffices if the adjacency condition $\left|h\left(s_{0}\right)-h\left(s_{1}\right)\right|=1$ was checked over all $c$ for all pairs $s_{0}$ and $s_{1}$ in $A$ corresponding to the same $c$. It remains to show that checking the situations where at least one $s_{i}$ is a sink (in their respective $A_{i}$ ) is enough.

Suppose we had a situation where checking just these pairs was not enough. This means for all pairs of vertices corresponding to the same $c$ (where at least one vertex is a sink in its respective $A_{i}$ ) we meet the adjacency condition, but for some such pair where neither are sinks, we have $\left|h\left(s_{0}\right)-h\left(s_{1}\right)\right| \neq 1$. Let $\left(s_{0}^{(0)}=s_{0}, s_{1}^{(0)}=s_{1}\right)$ be such a pair. Without loss of generality, assume $h\left(s_{0}\right)>h\left(s_{1}\right)$. Since $s_{0}$ is not a sink in $A_{0}$, there is some $s_{0}^{(1)}$ covering $s_{0}^{(0)}$ by an edge with some color $i \neq n$. Similarly, let $s_{1}=s_{1}^{(0)}$ be connected to $s_{1}^{(1)}$ via color $i$. Continuing this process, we must eventually come to a pair of vertices $s_{0}^{(l)}$ and $s_{1}^{(l)}$ where at least one is a sink. However, $h\left(s_{0}^{(l)}\right)=h\left(s_{0}\right)+l>h\left(s_{1}^{(l)}\right)$. Since we assumed that $h\left(s_{0}\right)>h\left(s_{1}\right)$ and $\left|h\left(s_{0}^{(l)}\right)-h\left(s_{1}^{(l)}\right)\right|=1$, the only way for these conditions to hold is if for each $i<l$, we had $h\left(s_{1}^{(i+1)}\right)=h\left(s_{1}^{(i)}\right)+1$. But this meant $\left|h\left(s_{0}^{(0)}\right)-h\left(s_{1}^{(0)}\right)\right|=1$ in the first place, a contradiction. Thus, these situations do not exist, and it suffices to only check pairs where at least one vertex is a sink.

Lemma 6.8 makes the following algorithm possible:

(1) For the data structure, represent all rankings $A$ by a set of $\operatorname{sinks} S(A)$ and their ranks as in Theorem 6.2 .

(2) Start with $R\left(I_{c}^{1}\right)$, which is a set of 2 rankings.

(3) Given a set of rankings in $R\left(I_{c}^{n-1}\right)$, iterate over all pairs of (possibly identical) rankings $(A, B)$ in $R\left(I_{c}^{n-1}\right) \times R\left(I_{c}^{n-1}\right)$. For each pair (with rank functions $h_{A}$ and $h_{B}$, respectively),

(a) Consider the ranking $B^{\prime}$ which is identical to $B$ except with the rank function $h_{B^{\prime}}(\overrightarrow{0})=h_{B}(\overrightarrow{0})+1$.

(b) For each sink $s \in S(A) \cup S\left(B^{\prime}\right)$, check that $\left|h_{A}(s)-h_{B^{\prime}}(s)\right|=1$.

(c) If the above is satisfied for all $s$, put $A \nearrow_{n} B^{\prime}$ in $R\left(I_{c}^{n}\right)$.

We used the above algorithm to compute the results for $n \leq 5$, which we include in Table 1 along with the counts of dashings (recall this is $o(n)=2^{2^{n}-1}$ ) and adinkras (which we obtain by multiplying $\left|R\left(I_{c}^{n}\right)\right|$ and $o(n)$, as the dashings and rankings are independent). Finding the answer for $n=6$ seems intractible with an algorithm that is at least linear in the number of solutions.

For chromotopologies other than $R\left(I_{c}^{n}\right)$ that can be decomposed (more generally, bipartite graphs of the form $A \times I)$, Lemma 6.8 still allows us to perform some similar computations. However, doing a case-by-case analysis for different graphs seems uninteresting without unifying rules. 
TABLE 1. Enumeration of dashings, rankings, and adinkras with chromotopology $I_{c}^{n}$.

\begin{tabular}{|l|l|l|l|}
\hline $\mathrm{n}$ & dashings & rankings & adinkras \\
\hline 1 & 2 & 2 & 4 \\
2 & 8 & 6 & 48 \\
3 & 128 & 38 & 4864 \\
4 & 32768 & 990 & 32440320 \\
5 & 2147483648 & 395094 & 848457904422912 \\
\hline
\end{tabular}

\section{Future DiRECTIONS}

The two main combinatorial problems we have extracted from the physics of adinkras are that of dashings and rankings of graphs. Theorem 5.10 gives a basically complete understanding of dashings for adinkraizable chromotopologies. However, it seems that the concept of dashings can be generalized to other graphs; it is strikingly similar to the concept of non-even graphs that arise in the study of Pfaffian graphs (see [26]). We are currently working on this generalization. Rankings are easier to generalize (they can be exactly reworded as graph homomorphisms into the infinite line graph) and should be fairly difficult to understand completely. In an upcoming work [20], we make some progress on exact enumeration and asymptotics of rankings using techniques of generating functions and chromatic polynomials.

We end this paper with some problems more relevant to the original physics context. We survey the recent papers and make some observations that would hopefully be useful to a reader interested in these problems. We believe each of these topics is a resource for rich mathematical discussion.

7.1. Constructing representations. Take an adinkra $A$, and consider the component fields (the bosons $\phi$ and the fermions $\psi$ ) as a basis. Then, consider a set of matrix generators $\left\{\rho\left(Q_{i}\right)\right\}$ in that basis, where $\rho\left(Q_{i}\right)$ is the adjacency matrix of the subgraph of $A$ induced by the edges of color $i$. If we order all the $\phi$ to come before all the $\psi$ in the row/column orderings, these matrices are block-antisymmetric of the form

$$
\rho\left(Q_{i}\right)=\left(\begin{array}{cc}
0 & L_{i} \\
R_{i} & 0
\end{array}\right)
$$

where the $L_{i}$ and $R_{i}$ are [15]'s garden matrices. For the adinkra in Figure 13, we have the following matrices, where the row/column indices are in the order 00, 11, 10, 01:

$$
\rho\left(Q_{1}\right)=\left(\begin{array}{cccc}
0 & 0 & 1 & 0 \\
0 & 0 & 0 & 1 \\
1 & 0 & 0 & 0 \\
0 & 1 & 0 & 0
\end{array}\right), \quad \rho\left(Q_{2}\right)=\left(\begin{array}{cccc}
0 & 0 & 0 & 1 \\
0 & 0 & -1 & 0 \\
0 & -1 & 0 & 0 \\
1 & 0 & 0 & 0
\end{array}\right) .
$$

So far, we have encoded the graph and the dashing into the matrices, but we do not yet have a representation of the supersymmetry algebra $\mathfrak{p o}^{1 \mid N}$. In fact, the $\rho\left(Q_{i}\right)$ form a representation of the Clifford algebra $\mathbf{C l}(N)$, which we discuss further in Section 7.3. The missing information (up to scalars) is the ranks of the vertices, which we can add into these matrices by adding the Hamiltonian operator $H$ 's to appropriate entries (recall Section 3 for details). In this sense, we are partitioning 


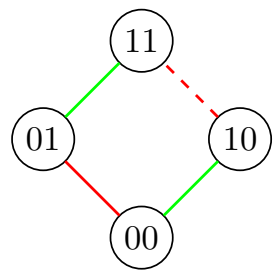

FiguRE 13. An adinkra with chromotopology $I^{2}$.

the infinite-dimensional basis of the representation into finite-dimensional "slices", each slice corresponding to a single finite-dimensional representation corresponding to our finite-dimensional matrices.

An obvious question to consider whenever we study representations is the following:

Question 7.1. Which adinkraic representations are irreducible?

In the valise case, this is well-understood (see [9]), with a surprising answer. If $L$ were not a maximal subspace inside $\mathbf{Z}_{2}^{n}$, we may quotient $I_{c}^{n} / L$ further to give a subrepresentation. Thus, irreducible valise adinkraic representations must have maximal doubly-even codes, which are self-dual. There seems to be no good general method for other rankings. The intuition of the obstruction is that this method of creating subrepresentations requires the vertices in each coset to come from the same rank, corresponding to the same engineering dimension. This kind of physics constraint is intricately connected to the selection of the right notion of isomorphism for adinkraic representations, which we now discuss.

7.2. When are two adinkras isomorphic? The instinct for the choice of the definition of "isomorphism" seems to be completely intuitive for the authors of the literature (see [15] and [10]), but we think it is important to have a formal discussion since the existing literature is somewhat cavalier about these definitions. Since the relevant discussions may be distracting, we compromise by moving much of them to Appendix B

Question 7.2. What is the right definition of "isomorphism" for two adinkraic representations? How does it relate to the combinatorics of adinkras?

- Following physics requirements, we define two adinkras $A$ and $B$ to be isomorphic if there is some matrix $R$ that transforms each generator $\rho\left(Q_{I}\right)$ of $A$ to the corresponding $\rho\left(Q_{I}\right)$ in $B$ via conjugation, with the stipulation that such a conjugation preserves the ranks of the component fields. To be explicit, let the component fields be partitioned into $P_{1} \cup P_{2} \cup \cdots$, where each $P_{i}$ contains all $\psi_{j}$ or $\phi_{j}$ of some rank (equivalently, engineering dimension). We require $M$ to be block-diagonal with respect to this partition.

- Following combinatorial intuition, we define two adinkras to be $C$-isomorphic if there is a sequence from one to the other via only vertex switchings or ranked poset isomorphisms. The discussion in Appendix $\mathrm{B}$ shows that both of these operations preserve isomorphism, so $C$-isomorphism is more restrictive than isomorphism. 
In a perfect world, these two notions would coincide. We are not so lucky here: the underlying graph is an invariant of the operations in the definition of the $C$ isomorphism, but there are adinkras with different graphs that correspond to isomorphic representations [8]. We still do not have a complete picture of the nuances between the two definitions. $C$-isomorphism is studied in more detail in [10] (where it is simply called an "isomorphism"; this is an example of why a formal discussion would be good to avoid accidental overlap of different concepts), which gives a deterministic algorithm to determine if two adinkras are $C$-isomorphic. Similar discussion relevant to isomorphism (even though it was not defined as such) can be found in [15] and [9].

A lot of interesting mathematics remain in the area. As an example, both [15] and [10] distinguish adinkras with the help of what amounts to the trace of the matrix

$$
\rho\left(Q_{1}\right) \rho\left(Q_{2}\right) \cdots \rho\left(Q_{N}\right)
$$

after multiplying by the matrix $\left(\begin{array}{cc}I & 0 \\ 0 & -I\end{array}\right)$. We would like to point out that this is precisely the well-known supertrace from the theory of superalgebras.

Finally, we can rephrase our notion of isomorphism with the language of quivers. Our isomorphism classes are exactly isomorphism classes of quiver representations with each node corresponding to one of the partitions $P_{i}$. For example, a valise representation (which corresponds to a 2-partition) for $I_{c}^{2}$ can be identified with the quiver from Figure 14, where the 2 nodes correspond to the bipartition $B \cup F$ of bosons and fermions. Each pair of edges of a color, say $i$, corresponds to the offdiagonal block matrices $M$ and $M^{\prime}$ that arise when we write each $Q_{i}$ as $\left(\begin{array}{cc}0 & M \\ M^{\prime} & 0\end{array}\right)$ corresponding to the bipartition. In general, if $B$ is further partitioned into $m_{1}$ parts and $F$ into $m_{2}$ parts, we would obtain $\left(m_{1}+m_{2}\right)$ vertices in total and $2 m_{1} m_{2}$ edges of each color. Sadly, the main value of this viewpoint may just be to suggest that a full understanding of the representation-theoretic nature of this problem is intractible, as the quivers all seem to be wild.

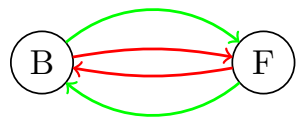

Figure 14. The quiver corresponding to a valise adinkra for $I_{c}^{2}$.

7.3. Clifford representations. In Section 3, we called the adinkraic representations arising from valise adinkras "Clifford supermultiplets". This is no big surprise - when we ignore the Hamiltonian $H$ in the defining relations

$$
\left\{Q_{I}, Q_{J}\right\}=2 \delta_{I J} H,
$$

we get precisely the Clifford algebra relations

$$
\left\{Q_{I}, Q_{J}\right\}=2 \delta_{I J}
$$

In other words, when we forget about the rank of an adinkra and look at only the well-dashed chromotopology (alternatively, the valise, where no bosons or fermions are privileged by rank from the other fields of the same type), we are really looking 
at a Clifford algebra representation, something that we saw in 7.1 and in the proof of Theorem 4.5. Therefore, we can think of adinkraic representations as extensions of representations of the Clifford algebra; 6] makes this analogy more rigorous by realizing adinkraic representations as filtered Clifford supermodules.

While Clifford algebras and their representations are well-understood (see [1] or [21]), the following is a natural question to ask:

Question 7.3. Can adinkras give us better intuition (organizational or computational) about the theory of Clifford representations?

In [9], each valise adinkra with the $I_{c}^{n}$ chromotopology is used to explicitly construct a representation of the Clifford algebra $\mathbf{C l}(n)$. This introduces a plethora of representations with lots of isomorphisms between them - after all, there are at most 2 irreducible representations for each Clifford algebra over $\mathbf{R}$. Even for a beginner, the visually appealing aspect of adinkras (more precisely, well-dashed chromotopologies) may be the foundation of an easier mental model of thinking about Clifford algebras.

7.4. Extensions. As we brushed over in Section 3, the adinkraic representations correspond to the 1-dimensional (more precisely, $(1,0)$-dimensional) worldline situation with $N$ supercharge generators. We now discuss the more general context. Helpful expositions of related concepts are [14] and [27].

In general, we are interested in some $(1+q)$-dimensional vector space over $\mathbf{R}$ with Lorentzian signature $(1, q)$. Besides our $(1,0)$ situation, some examples are $(1,1)$ (worldsheet) and $(1,3)$ (Lorentzian spacetime). We can write this more general situation as $(1, q \mid N)$-supersymmetry 2 We would then call the corresponding superalgebra $\mathfrak{p o}^{1+q \mid N}$, which specializes to the particular superalgebra $\mathfrak{p o}^{1 \mid N}$ we have been working with when $q=0$.

In the case where $(1+q)=2,6(\bmod 8)$, we actually get two different types of supercharge generators (this again corresponds to the fact that there are two Clifford algebra representations over $\mathbf{R}$ in those situations), so we can partition $N=P+Q$ and call these situations $(1, q \mid P, Q)$-supersymmetry.

Question 7.4. What happens when we look at $q>0$ ? What kind of combinatorial objects appear? Will the machinery we developed for adinkraic representations in the wordline case be useful?

[16] examines the $(1,1)$-case, where the combinatorics get more complex. The reader may have gotten the intuition that the dashings and rankings are fairly independent conditions of the adinkra. This is true for the $(1,0)$-case but no longer holds for the $(1,1)$-case, where certain forbidden patterns arise that depend both on the dashings and rankings. 18] creates $(1,1 \mid P, Q)$ representations by tensoring and quotienting worldline representations, similar in spirit to the construction of representations of semisimple Lie algebras.

In a different direction, [12] and [13] examine which worldline representations can be "shadows" of higher-dimensional ones and give related consistency-tests and

\footnotetext{
${ }^{2}$ Here is another unfortunate source of language confusion: for physicists, $N$ means the number of supersymmetry generators, whereas mathematicians would instead count the total number of dimensions and write $d N$ instead of $N$, where $d$ is the real dimension of the minimal spin- $(1 / 2)$ representation of $\mathbf{R}^{1+q}$. These minimal dimensions are $1,1,2,4,8, \ldots$ starting with $n=1$. Luckily, for most of this paper, $d=1$ and we have no problems. A clear explanation is given in [14.
} 
algorithms. As worldline representations are involved, the 1-dimensional theory already built plays an instrumental role.

\section{Appendix A. Clifford algebras}

The Clifford algebra is an algebra $\mathbf{C l}(n)$ with generators $\gamma_{1}, \ldots, \gamma_{n}$ and the anticommutation relations

$$
\left\{\gamma_{i}, \gamma_{j}\right\}=2 \delta_{i, j} \cdot 1
$$

The Clifford algebra can be defined for any field, but we will typically assume $\mathbf{R}$. There are also more general definitions than what we give, though we won't need them for our paper. For references, see 1 or 4 .

We can associate an element of the Clifford algebra to any $n$-codeword $b=$ $b_{1} b_{2} \cdots b_{n}$, by defining

$$
\operatorname{clif}(b)=\prod_{i} \gamma_{i}^{b_{i}}
$$

where the product is taken in increasing order of $i$. Call these elements monomials. The $2^{n}$ possible monomials form a basis of $\mathbf{C l}(n)$ as a vector space, and the $2^{n+1}$ signed monomials $\pm \operatorname{clif}(b)$ form a multiplicative group $\operatorname{SMon}(n)$, or just SMon when the context is clear. It is easy to see that two signed monomials of degrees $a$ and $b$ commute if and only if $a b=0(\bmod 2)$, and one could equivalently define Clifford algebras as commutative superalgebras with odd and even parts generated by the odd and even degree monomials, respectively.

The following facts are needed for Proposition 4.1, where we defined the notion of an almost doubly-even code as a code with all codewords having weight 0 or 1 $(\bmod 4)$.

Lemma A.1. For any two codewords $w_{1}$ and $w_{2}$ in an almost doubly-even code, we have

$$
\left(w_{1} \cdot w_{2}\right)+\operatorname{wt}\left(w_{1}\right) \operatorname{wt}\left(w_{2}\right)=0 \quad(\bmod 2),
$$

where the first term is the dot product in $\mathbf{Z}_{2}^{n}$.

Proof. Given an almost doubly-even length $n$ code $L$, introduce 3 new bits in the code to construct a doubly-even length $(n+3)$ code $L^{\prime}$ via the following map $g$ : for $w \in L$, let $g(w \in L)$ be the concatenation $w \mid 111$ if $w$ has odd weight and $w \mid 000$ if $w$ has even weight. Since the weight parity of two codewords are additive modulo $2, g(v+w)=g(v)+g(w)$ and $L^{\prime}$ is linear. Our construction also clearly ensures that $L^{\prime}$ is doubly-even. It is well-known (see, for example, [19]) that doubly-even codes are self-orthogonal, so $\left(g\left(w_{1}\right) \cdot g\left(w_{2}\right)\right)=0(\bmod 2)$ for all $w_{1}$ and $w_{2}$ in $L$. But $\left(g\left(w_{1}\right) \cdot g\left(w_{2}\right)\right)-\left(w_{1} \cdot w_{2}\right)$ is $0(\bmod 2)$ when either $w_{1}$ or $w_{2}$ has even weight (because the additional bits 000 cannot affect the dot product) and is $1(\bmod 2)$ exactly when both $w_{1}$ and $w_{2}$ have odd weights. This is equivalent to the condition we want to prove.

Lemma A.2. The image of a code $L$ under clif is commutative if and only if for all $a, b \in L$,

$$
(a \cdot b)+\operatorname{wt}(a) \operatorname{wt}(b)=0 \quad(\bmod 2) .
$$

In particular, an almost doubly-even code satisfies this property. 
Proof. Finally, consider $\operatorname{clif}(a)=\gamma_{a_{1}} \ldots \gamma_{a_{r}}$ and $\operatorname{clif}(b)=\gamma_{b_{1}} \ldots \gamma_{b_{s}}$, where $r=$ $\operatorname{wt}(a)$ and $s=\operatorname{wt}(b)$. Note we can get from $\operatorname{clif}(a) \operatorname{clif}(b)$ to $\operatorname{clif}(b) \operatorname{clif}(a)$ in $\operatorname{wt}(a) \operatorname{wt}(b)$ transpositions, where we move, in order $\gamma_{b_{1}}, \cdots, \gamma_{b_{s}} \operatorname{through} \operatorname{clif}(a)$ to the left, picking up exactly wt $(a)$ powers of $(-1)$. However, we've also overcounted once for each time $a$ and $b$ shared a generator $\gamma_{i}$, since $\gamma_{i}$ commutes with itself. Therefore, we have exactly $(a \cdot b)+\mathrm{wt}(a) \mathrm{wt}(b)$ powers of $(-1)$. The condition for commutativity is then that this quantity be even for all pairs $a$ and $b$.

Proposition A.3. A code $L$ is an almost doubly-even code if and only if $L$ has the property that for a suitable sign function $s(v) \in\{ \pm 1\}$ with $s(\overrightarrow{0})=1$, the set $\mathbf{S M o n}_{L}=\{s(v) \operatorname{clif}(v) \mid v \in L\}$ forms a subgroup of SMon.

Proof. Without loss of generality, say $\operatorname{clif}(v)=s(v) \prod_{i=1}^{k} \gamma_{i}$. Then

$$
\begin{aligned}
\operatorname{clif}(v)^{2} & =\left(\gamma_{1} \gamma_{2} \cdots \gamma_{k}\right)\left(\gamma_{1} \gamma_{2} \cdots \bar{q}_{k}\right) \\
& =(-1)^{(k-1)}\left(\gamma_{2} \gamma_{3} \cdots \gamma_{k}\right)\left(\gamma_{1}\right)\left(\gamma_{1} \gamma_{2} \cdots \gamma_{k}\right) \\
& =(-1)^{k(k-1) / 2} .
\end{aligned}
$$

Suppose $s$ exists. Then, we must not have $(-1) \in \mathbf{S M o n}_{L}$ ( since we already have $\left.1 \in \mathbf{S M o n}_{L}\right)$. Therefore, it is necessary to have the last quantity equal 1 , which happens exactly when $\operatorname{wt}(v)=0$ or $1(\bmod 4)$ for all $v \in L$.

If $L$ were an almost doubly-even code, then pick a basis $l_{1}, \ldots, l_{k}$ of $L$ and assign $s\left(l_{i}\right)=1$ for all $i$. Note by the above equations that $\operatorname{clif}\left(l_{i}\right)^{2}=1$ for all $i$. The linear independence of the $l_{i}$ is equivalent to the condition that no group axioms are broken by this choice of $l_{i}$. Now, we can extend the definition to

$$
s\left(\prod_{i \in I} \operatorname{clif}\left(l_{i}\right)\right)=\prod_{i \in I} s\left(\prod\left(\operatorname{clif}\left(l_{i}\right)\right)\right)
$$

which is well-defined and closed under multiplication since the $l_{i}$ commute and square to 1 .

\section{Appendix B. Some discussion on Adinkra isomorphisms}

We usually consider two representations as isomorphic if they are conjugate by some change of basis. However, because of our physics context we need more restrictions. To find the right notion, we now recall/define three types of transformations and discuss what it means for them to give the "same" adinkra.

- Recall that a vertex switching changes the dashing of all edges adjacent to a vertex. This corresponds to simply changing the sign (as a function) of the component field corresponding to that vertex, or equivalently, conjugation of the representation by a diagonal matrix of all 1's except for a single $(-1)$. It is reasonable to consider this move as an operation that preserves isomorphism.

- Let a color permutation permute the names of the colors (in the language of codes, it is a simultaneous column permutation of the codewords corresponding to each vertex). In our situation, this is just a shuffling of the generators, so at first glance it is reasonable to consider this operation to preserve isomorphism. The existing literature, e.g. 9], seems to do so as well. However, this is not quite what we want in a natural definition, where we need to consider the base ring fixed. By analogy, consider the $k[x, y]$ modules $k[x, y] /(x)$ and $k[x, y] /(y)$, which may look "equivalent" (they are 
indeed isomorphic as algebras) but are not isomorphic as modules. They should not be: we really want $A \oplus B$ to be isomorphic to $A \oplus B^{\prime}$ if $B$ and $B^{\prime}$ were isomorphic, but the direct sums $k[x, y] /(x) \oplus k[x, y] /(x)$ and $k[x, y] /(x) \oplus k[x, y] /(y)$ are not isomorphic in any reasonable way.

In fact, the existing adinkra literature notices this problem when considering disconnected adinkras (i.e. adinkras with topology of a disconnected graph). These graphs correspond to direct sums of representations of single adinkras. However, since a color permutation is done over all disjoint parts simultaneously, if we consider color permutations as operations that preserve isomorphism, we obtain situations where $A \cong C$ and $B \cong D$, but $A \oplus B \neq C \oplus D$. The literature deals with this situation by calling color permutations outer isomorphisms. We believe the correct thing to do is to just not consider these situations isomorphic and treat them as a separate kind of similarity.

- Let a vertex permutation permute the vertex labels of an adinkra $A$. This corresponds to conjugating the matrices $\rho\left(Q_{i}\right)$ by permutation matrices. Here it makes sense to impose further physics constraints: we want these transformations to preserve the engineering dimensions of the component fields. This prevents us from allowing arbitrary vertex permutations and simply considering two adinkraic representations isomorphic if they're conjugate. On the adinkras side, this corresponds to us enforcing that the rank function of $A$ be preserved under any vertex permutation (in particular, bosons must go to bosons, and fermions to fermions). Happily, this neatly corresponds to the natural definition of isomorphism for ranked posets.

\section{ACKNOWLEDGEMENTS}

First and foremost, we thank Sylvester "Jim" Gates for teaching the subject to us. We thank Brendan Douglas, Greg Landweber, Kevin Iga, Richard Stanley, Joel Lewis, and Steven Sam for helpful discussions. Anatoly Preygel and Nick Rozenblyum gave us very enlightening lessons in algebraic topology and made the relevant sections possible. Alexander Postnikov made the observation about quivers. We are especially grateful to Tristan Hübsch for his unreasonably generous gift of time and patience through countless communications.

The author was supported by an NSF graduate research fellowship.

\section{REFERENCES}

[1] M. F. Atiyah, R. Bott, and A. Shapiro, Clifford modules, Topology 3 (1964), no. suppl. 1, 3-38. MR0167985 (29 \#5250)

[2] I. N. Bernštern, I. M. Gel'fand, and S. I. Gel'fand, Differential operators on the base affine space and a study of $\mathfrak{g}$-modules, Lie groups and their representations (Proc. Summer School, Bolyai János Math. Soc., Budapest, 1971), Halsted, New York, 1975, pp. 21-64. MR0578996 (58 \#28285)

[3] Raoul Bott and Loring W. Tu, Differential forms in algebraic topology, Graduate Texts in Mathematics, vol. 82, Springer-Verlag, New York, 1982. MR658304 (83i:57016)

[4] Pierre Deligne, Notes on spinors, (Princeton, NJ, 1996/1997), Amer. Math. Soc., Providence, RI, 1999, pp. 99-135. MR1701598(2001c:81059)

[5] C. F. Doran, M. G. Faux, S. J. Gates, Jr., T. Hubsch, K. M. Iga, and G. D. Landweber, An application of cubical cohomology to adinkras and supersymmetry representations. In preparation. 
[6] C. F. Doran, M. G. Faux, S. J. Gates, Jr., T. Hubsch, K. M. Iga, and G. D. Landweber, Off-shell supersymmetry and filtered Clifford supermodules, March 2006. arXiv:math-ph/0603012.

[7] C. F. Doran, M. G. Faux, S. J. Gates Jr., T. Hübsch, K. M. Iga, and G. D. Landweber, On graph-theoretic identifications of Adinkras, supersymmetry representations and superfields, Internat. J. Modern Phys. A 22 (2007), no. 5, 869-930, DOI 10.1142/S0217751X07035112. MR2311874(2008h:81163)

[8] C. F. Doran, M. G. Faux, S. J. Gates, Jr., T. Hübsch, K. M. Iga, G. D. Landweber, and R. L. Miller, Adinkras for Clifford Algebras, and Worldline Supermultiplets, November 2008. arXiv:hep-th/0811.3410.

[9] C.F. Doran, M.G. Faux, Jr. Gates, S.J., T. Hübsch, K.M. Iga, et al., Codes and Supersymmetry in One Dimension, 2011. arXiv:hep-th/1108.4124.

[10] B. L. Douglas, S. J. Gates, Jr., and J. B. Wang, Automorphism Properties of Adinkras, September 2010. arXiv: hep-th/1009.1449.

[11] M. G. Faux and S. J. Gates, Jr., Adinkras: A graphical technology for supersymmetric representation theory. PHYS.REV.D, 71:065002, 2005. arXiv:hep-th/0408004.

[12] M. G. Faux, K. M. Iga, and G. D. Landweber, Dimensional Enhancement via Supersymmetry, July 2009 .

[13] M. G. Faux and G. D. Landweber, Spin holography via dimensional enhancement. Physics Letters B, 681:161-165, October 2009.

[14] Daniel S. Freed, Five lectures on supersymmetry, American Mathematical Society, Providence, RI, 1999. MR1707282(2000h:58015)

[15] S. J. Gates, Jr., J. Gonzales, B. Mac Gregor, J. Parker, R. Polo-Sherk, V. G. J. Rodgers, and L. Wassink. 4D, $\mathcal{N}=1$ supersymmetry genomics (I). Journal of High Energy Physics, 12, December 2009.

[16] S. J. Gates, Jr. and T. Hubsch, On Dimensional Extension of Supersymmetry: From Worldlines to Worldsheets, April 2011.

[17] Allen Hatcher, Algebraic topology, Cambridge University Press, Cambridge, 2002. MR.1867354(2002k:55001)

[18] T. Hubsch, Weaving Worldsheet Supermultiplets from the Worldlines Within, April 2011. arXiv:hep-th/1104.3135.

[19] W. Cary Huffman and Vera Pless, Fundamentals of error-correcting codes, Cambridge University Press, Cambridge, 2003. MR.1996953 (2004k:94077)

[20] A. Klein and Y. X. Zhang, Enumerating graded poset structures on graphs. In preparation.

[21] H. Blaine Lawson Jr. and Marie-Louise Michelsohn, Spin geometry, Princeton Mathematical Series, vol. 38, Princeton University Press, Princeton, NJ, 1989. MR1031992 (91g:53001)

[22] R. Miller. Doubly-even codes. http://www.rlmiller.org/de_codes/.

[23] Abdus Salam and J. Strathdee, Super-gauge transformations, Nuclear Phys. B76 (1974), 477-482. MR.0356737 (50 \#9206)

[24] J. J. Seidel, A survey of two-graphs (English, with Italian summary), Colloquio Internazionale sulle Teorie Combinatorie (Rome, 1973), Accad. Naz. Lincei, Rome, 1976, pp. 481-511. Atti dei Convegni Lincei, No. 17. MR0550136 (58 \#27659)

[25] Richard P. Stanley, Enumerative combinatorics. Vol. 1, Cambridge Studies in Advanced Mathematics, vol. 49, Cambridge University Press, Cambridge, 1997. With a foreword by Gian-Carlo Rota; corrected reprint of the 1986 original. MR.1442260 (98a:05001)

[26] Robin Thomas, A survey of Pfaffian orientations of graphs, International Congress of Mathematicians. Vol. III, Eur. Math. Soc., Zürich, 2006, pp. 963-984. MR2275714 (2008f:05161)

[27] V. S. Varadarajan, Supersymmetry for mathematicians: an introduction, Courant Lecture Notes in Mathematics, vol. 11, New York University Courant Institute of Mathematical Sciences, New York, 2004. MR2069561 (2005g:58011)

[28] Doug Wiedemann, A computation of the eighth Dedekind number, Order 8 (1991), no. 1, 5-6, DOI 10.1007/BF00385808. MR1129608 (92h:05003)

Department of Mathematics, Massachusetts Institute of Technology, Cambridge, MassachusetTs 02139

Current address: Department of Mathematics, University of California, Berkeley, Berkeley, California 94720 